\title{
ANALYSIS OF A FINITE PML APPROXIMATION TO THE THREE DIMENSIONAL ELASTIC WAVE SCATTERING PROBLEM
}

\author{
JAMES H. BRAMBLE, JOSEPH E. PASCIAK, AND DIMITAR TRENEV
}

\begin{abstract}
We consider the application of a perfectly matched layer (PML) technique to approximate solutions to the elastic wave scattering problem in the frequency domain. The PML is viewed as a complex coordinate shift in spherical coordinates which leads to a variable complex coefficient equation for the displacement vector posed on an infinite domain (the complement of the scatterer). The rapid decay of the PML solution suggests truncation to a bounded domain with a convenient outer boundary condition and subsequent finite element approximation (for the truncated problem).

We prove existence and uniqueness of the solutions to the infinite domain and truncated domain PML equations (provided that the truncated domain is sufficiently large). We also show exponential convergence of the solution of the truncated PML problem to the solution of the original scattering problem in the region of interest. We then analyze a Galerkin numerical approximation to the truncated PML problem and prove that it is well posed provided that the PML damping parameter and mesh size are small enough. Finally, computational results illustrating the efficiency of the finite element PML approximation are presented.
\end{abstract}

\section{INTRODUCTION}

In this paper, we consider techniques for approximating the solutions of frequency domain elastic wave scattering problems. These problems are posed on unbounded domains with a far field boundary condition given by the so-called Kupradze-Sommerfeld radiation condition. This condition prescribes two different Sommerfeld conditions on components of the solution at infinity. Specifically, away from the scatterer, the solution is broken up into a solenoidal and an irrotational component. These components satisfy Helmholtz equations with different wave numbers. The Kupradze-Sommerfeld radiation condition involves using Sommerfeld radiation conditions with the corresponding wave numbers on the separate components.

The fundamental challenge posed by this far field boundary condition is that it appears that one has to break up the field at the discrete level. One possible way to do this is to reformulate the problem in terms of potentials, keeping the solenoidal and irrotational components separate [9, 16. As the potentials satisfy

Received by the editor October 30, 2008 and, in revised form, July 15, 2009.

2010 Mathematics Subject Classification. Primary 65F10, 78M10, 65N30.

Key words and phrases. Elastic wave problem, Helmholtz equation, elastic waves scattering, PML layer. 
the same radiation conditions as their derivatives, classical numerical techniques for implementing Sommerfeld boundary conditions can be applied to the separate (but coupled) potential problems. There are, however, two significant drawbacks to this approach. First, the introduction of potentials adds complexity to the problem, especially in three dimensional applications where gauge conditions must be introduced for uniqueness. Second, the resulting field approximations are obtained by differentiation, and this often results in loss of approximation accuracy.

In this paper, we shall study a so-called perfectly matched layer (PML) reformulation of the elastic wave scattering problem. From an engineering point of view, PML can be thought of as the introduction of a fictitious material designed to absorb energy away from the region of interest (usually close to the scatterer). A perfectly matched layer is one which absorbs all energy sent into it without creating any reflected waves. In [1, Bérenger showed that for a half plane (and Maxwell's equations), such a layer could be constructed which was without reflection of incoming plane waves of any angle. His approach involved the introduction of additional variables and equations in the "ficticious material" region.

In [5], Chew and Weedon derived the PML model of Bérenger [1] in terms of complex coordinate stretching. This point of view was very important in that it allowed one to see that a properly formulated PML approximation could sometimes be constructed which preserved the solution in the region of interest while decaying exponentially at infinity. Because of this decay, the solution to the PML problem can be approximated by a problem on a finite domain (with convenient boundary condition) and subsequently approximated by numerical methods, e.g., the finite element method. The complex coordinate stretching point of view was further applied by Collino and Monk to derive PML models in rectangular and polar coordinates in [6] for acoustic problems. An analysis of the spherical PML equations for the three dimensional electromagnetic and acoustic problems (both on the infinite and truncated domains) was given by Bramble and Pasciak in [2], where the inf-sup conditions useful for finite element error analysis were proved.

Extensions of Bérenger's ideas for the elastic waves problem were considered by Hastings et al. in 9 and independently by Chew and Liu in 44. The PML model in [9] is based on a potential formulation of the elastic wave problem. Similar to the original Bérenger model, additional splitting of the fields was required. Another PML model based on the stress-velocity formulation of the problem was proposed by Collino and Tsogka in [7.

In this paper, we apply the complex shift technique in spherical coordinates to derive and analyze a PML model for the time-harmonic elastic wave problem. Similar to [2], the complex shift technique leads to a single (variable coefficient) equation for the displacement vector and, in contrast to the models in [7] and [9], requires no field splitting or additional unknowns. A PML model similar to ours was proposed in [20, and limited numerical results were reported.

Also in this paper, we define a PML layer in terms of a fixed parameter $\sigma_{0}$ and then allow the size of the computational domain to increase. We shall see, first, that this technique leads to a PML model on the infinite domain for the time-harmonic elastic wave problem with the desired properties, i.e., its solution coincides with the original on the domain of interest (surrounded by the PML layer) while decaying exponentially inside the layer. Moreover, it takes care of both wave components simultaneously without field splitting and only requires 
the introduction of special "PML" coefficients in the PML region. We then show that the solution of the truncated PML problem, although no longer equal to the original solution, converges exponentially to it (as the size of the computational domain increases) in the region of interest. To show stability of the truncated PML solution, we derive $H^{1}$-based inf-sup conditions.

As far as we know, the $H^{1}$-based inf-sup conditions cannot, in general, be developed using a lower order perturbation argument based on the lemmas of [15, 19]. Accordingly, the classical finite element analysis for non-coercive problems [18] cannot be applied. It is however possible to show that one can derive a stable approximation in terms of $H^{-1}$ least-squares, although we shall not do so here. In contrast, if we limit the size of the PML coefficient, then we shall see that it is possible to prove an inf-sup condition via perturbation, and classical finite element analysis implies convergence of the finite element approximation.

We note that all constants, except those in the finite element section, may depend on $\sigma_{0}$ (which we take as fixed) but are always independent of $R_{t}$, the size of the computational domain. We make no attempt to analyze PML strategies which involve simultaneously varying $\sigma_{0}$ and $R_{t}$. The constants appearing in the finite element section depend on the constants appearing in elliptic regularity "shift theorems." Although we believe that they too can be bounded independently of $R_{t}$, we have not included a proof. In any event, the regularity constant only effects the smallness of the approximation parameter $h$ required for stability and approximation of the finite element method. The numerical results show that this is not a problem in practice.

The outline of the remainder of this paper is as follows. In Section 2, we formulate the elastic wave problem and state some properties of its solution. Section 3 introduces the PML viewed as a complex coordinate shift and derives some properties of the modified differential operators. Existence and uniqueness of the infinite domain PML problem and the truncated PML problem are shown in Sections 4 and 5. A convergence result for the solution of the truncated PML problem is also given in Section 5. Section 6 deals with the numerical approximation of the truncated PML problem by finite elements. Finally, the results of numerical experiments illustrating the behavior of the method on a model two dimensional problem are given in Section 7.

\section{Formulation of the elastic WaVe Problem}

Throughout this paper, we shall have to deal with complex valued functions in various Sobolev spaces. For a domain $D$, let $L^{2}(D)$ be the space of complex valued functions whose absolute value is square integrable on $D$ and let $\boldsymbol{L}^{2}(D)=$ $\left(L^{2}(D)\right)^{3}$ be the space of vector valued functions whose components are in $L^{2}(D)$. We shall use $(\cdot, \cdot)_{D}$ to denote the (vector or scalar Hermitian) $L^{2}(D)$-inner product (sesquilinear). The scalar and vector Sobolev spaces on $D$ will be denoted $H^{s}(D)$ and $\boldsymbol{H}^{s}(D)$, respectively. Sobolev spaces with vanishing boundary conditions are denoted by $H_{0}^{s}(D)$ or $\boldsymbol{H}_{0}^{s}(D)$ and can be characterized as the completion of $C_{0}^{\infty}(D)$ or $\boldsymbol{C}_{0}^{\infty}(D)$ (respectively) under the corresponding Sobolev norms. We shall use bold symbols to denote vector valued functions and operators. When the inner product is on $\Omega^{c}$ (defined below), we will use the simpler notation $(\cdot, \cdot)$. All forms will be sesquilinear unless otherwise noted. 
In this section, we formulate the elastic wave problem and its far field boundary condition. Let $\Omega$ be a bounded domain, with boundary $\Gamma$, containing the origin, and let $\Omega^{c}$ denote its complement. We seek a vector valued function $\boldsymbol{u} \in \boldsymbol{H}_{l o c}^{1}\left(\Omega^{c}\right)$ satisfying

$$
\Delta \boldsymbol{u}+\gamma \nabla \nabla \cdot \boldsymbol{u}+k^{2} \boldsymbol{u}=\mathbf{0} \text { in } \Omega^{c}
$$

and

$$
\boldsymbol{u}=\boldsymbol{g} \text { on } \Gamma \text {. }
$$

Here $\gamma$ and $k$ are positive real numbers and $\boldsymbol{g}$ is given in $\boldsymbol{H}^{1 / 2}(\Gamma)$.

The formulation is completed by imposing the "so-called" Kupradze-Sommerfeld far field radiation condition. This condition involves decomposing the function $\boldsymbol{u}$ away from $\Omega$ as

$$
\boldsymbol{u}=\boldsymbol{\zeta}+\boldsymbol{\psi},
$$

with $\zeta$ solenoidal and $\psi$ irrotational. As shown in 3 , the components can be chosen to satisfy Helmholtz equations,

$$
\Delta \boldsymbol{\zeta}+k^{2} \boldsymbol{\zeta}=\mathbf{0}
$$

and

$$
\Delta \psi+k_{1}^{2} \boldsymbol{\psi}=\mathbf{0}
$$

where $k_{1}=k / \sqrt{1+\gamma}$. The Kupradze-Sommerfeld radiation condition imposes the corresponding Sommerfeld radiation conditions on the components, i.e.,

$$
\lim _{r \rightarrow \infty} r\left(\frac{\partial \boldsymbol{\zeta}}{\partial n}-i k \boldsymbol{\zeta}\right)=\mathbf{0}
$$

and

$$
\lim _{r \rightarrow \infty} r\left(\frac{\partial \boldsymbol{\psi}}{\partial n}-i k_{1} \boldsymbol{\psi}\right)=\mathbf{0}
$$

Let $B_{R}$ denote the open ball of radius $R$ centered at the origin and assume that $\bar{\Omega}$ is contained in $B_{R}$. The above decomposition is, in fact, uniquely determined from the values of $\boldsymbol{u}$ on $B_{R}$ (see, e.g., [3]).

Since the components above satisfy Helmholtz equations and Sommerfeld boundary conditions, they can be expanded in series outside of $B_{R}$. Indeed,

$$
\boldsymbol{\psi}(\boldsymbol{x})=\sum_{n=0}^{\infty} \sum_{|m| \leq n} \gamma_{n, m} p_{n}(r) Y_{n, m}(\hat{\boldsymbol{x}}),
$$

with $\gamma_{n, m} \in \mathbb{R}^{3}$. Here $p_{n}(r) \equiv h_{n}^{(1)}\left(k_{1} r\right), h_{n}^{(1)}$ is the Hankel function of the first kind of order $n, Y_{n, m}$ are spherical harmonics, $r=|\boldsymbol{x}|$ and $\hat{\boldsymbol{x}}=\boldsymbol{x} / r$. Similarly,

$$
\boldsymbol{\zeta}=\sum_{n=0}^{\infty} \sum_{|m| \leq n} \boldsymbol{\alpha}_{n, m} q_{n}(r) Y_{n, m}(\hat{\boldsymbol{x}})
$$

with $\boldsymbol{\alpha}_{n, m} \in \mathbb{R}^{3}$ and $q_{n}(r) \equiv h_{n}^{(1)}(k r)$. Hence outside of $B_{R}, \boldsymbol{u}$ may be expanded in a series of the form

$$
\boldsymbol{u}=\sum_{n=0}^{\infty} \sum_{|m| \leq n}\left(\boldsymbol{\alpha}_{n, m} q_{n}(r)+\boldsymbol{\gamma}_{n, m} p_{n}(r)\right) Y_{n, m}(\hat{\boldsymbol{x}}) .
$$


As is well known [8], the above series and all of its derivatives converge uniformly on compact subsets in the exterior of $\bar{R}$.

The following theorem, which will be essential for this paper, is the main result of 3 .

Theorem 2.1. For any function $\boldsymbol{g}$ in $\boldsymbol{H}^{1 / 2}(\Gamma)$, there is a unique solution $\boldsymbol{u}$ in $\boldsymbol{H}_{l o c}^{1}\left(\Omega^{c}\right)$ to the elastic wave problem (2.1), satisfying conditions (2.2), (2.4) and (2.5). Moreover, for any $R, \boldsymbol{u} \in \boldsymbol{H}^{1}\left(\Omega^{c} \cap B_{R}\right)$ and satisfies

$$
\|\boldsymbol{u}\|_{1,\left(\Omega^{c} \cap B_{R}\right)} \leq C(R)\|\boldsymbol{g}\|_{1 / 2, \Gamma} .
$$

\section{PML OPERATORS}

Throughout this paper, we shall use a sequence of finite subdomains of $\Omega^{c}$ with spherical outer boundaries. Let $r_{-1}<r_{0}<r_{1}<r_{2}$ be an increasing sequence of positive real numbers and let $\Omega_{i}$ denote (the interior of) the open ball $B_{i}$ of radius $r_{i}$ excluding $\bar{\Omega}$ (we assume that $r_{-1}$ is large enough so that the corresponding ball contains $\bar{\Omega}$ ). We denote the outer boundary of $\Omega_{i}$ by $\Gamma_{i}$. The values of $r_{-1}, r_{0}, r_{1}$ are independent of the computational outer boundary scaling parameter $R_{t}$ (introduced below).

The differential operators involved in PML approximations can be defined in terms of a formal complex change of variables (or stretching). In this paper, we consider a "change of variables" of the form

$$
T \boldsymbol{x}=(1+i \widetilde{\sigma}(r)) \boldsymbol{x},
$$

where $r=|\boldsymbol{x}|$ and $\boldsymbol{x} \in \mathbb{R}^{3}$.

Following [10, we use a function $\widetilde{\sigma}$ which results in a constant coefficient problem outside the transition. Given $\sigma_{0}, r_{0}$, and $r_{1}$, we start with a function $\widetilde{\sigma} \in C^{2}\left(\mathbb{R}^{+}\right)$ satisfying

$$
\begin{aligned}
& \tilde{\sigma}(r)=0 \quad \text { for } r \leq r_{0}, \\
& \widetilde{\sigma}(r)=\sigma_{0} \text { for } r \geq r_{1}, \\
& \widetilde{\sigma}(r) \text { increasing for } r \in\left(r_{0}, r_{1}\right) .
\end{aligned}
$$

One obvious construction of such a function $\widetilde{\sigma}$ in the transition layer $r_{0} \leq r \leq r_{1}$ with the above properties is given by the fifth order polynomial,

$\tilde{\sigma}(r)=\sigma_{0}\left(\int_{r_{0}}^{r}\left(t-r_{0}\right)^{2}\left(r_{1}-t\right)^{2} d t\right)\left(\int_{r_{0}}^{r_{1}}\left(t-r_{0}\right)^{2}\left(r_{1}-t\right)^{2} d t\right)^{-1}$ for $r_{0} \leq r \leq r_{1}$.

A smoother $\widetilde{\sigma}$ can be constructed by increasing the exponents in the above formula.

Let $\widetilde{d}(r)=1+i \widetilde{\sigma}(r), d(r)=1+i \sigma(r), \sigma(r)=\widetilde{\sigma}(r)+r \widetilde{\sigma}^{\prime}(r)$, and

$$
\boldsymbol{P}=\frac{1}{r^{2}}\left(\begin{array}{ccc}
x_{1}^{2} & x_{1} x_{2} & x_{1} x_{3} \\
x_{1} x_{2} & x_{2}^{2} & x_{2} x_{3} \\
x_{1} x_{3} & x_{2} x_{3} & x_{3}^{2}
\end{array}\right) .
$$

We note that $\boldsymbol{P}$ is the orthogonal projection along the vector $\boldsymbol{x}$. We introduce the following matrices (which depend on $\boldsymbol{x} \in \mathbb{R}^{3}$ ):

$$
\begin{array}{r}
\boldsymbol{A u}=\frac{1}{\widetilde{d}^{2}} u_{r} \boldsymbol{e}_{r}+\frac{1}{d \widetilde{d}}\left(u_{\phi} \boldsymbol{e}_{\phi}+u_{\theta} \boldsymbol{e}_{\theta}\right)=\frac{1}{\widetilde{d}^{2}} \boldsymbol{P} \boldsymbol{u}+\frac{1}{d \widetilde{d}}(\boldsymbol{I}-\boldsymbol{P}) \boldsymbol{u}, \\
\boldsymbol{B} \boldsymbol{u}=d u_{r} \boldsymbol{e}_{r}+\widetilde{d}\left(u_{\phi} \boldsymbol{e}_{\phi}+u_{\theta} \boldsymbol{e}_{\theta}\right)=d \boldsymbol{P} \boldsymbol{u}+\widetilde{d}(\boldsymbol{I}-\boldsymbol{P}) \boldsymbol{u} .
\end{array}
$$

In the above, $(r, \phi, \theta)$ denote the spherical coordinates of $\boldsymbol{x}$. 
We apply a formal change of variable (see, e.g., [1]), and are led to PML operators:

$$
\begin{aligned}
\widetilde{\nabla} w & =\boldsymbol{B}^{-1} \boldsymbol{\nabla} w, \\
\widetilde{\Delta} & =\frac{1}{\widetilde{d}^{2} d} \nabla \cdot\left(\boldsymbol{A}^{-1} \boldsymbol{B}^{-1} \boldsymbol{\nabla}\right), \\
\widetilde{\boldsymbol{\Delta}} \boldsymbol{w} & =\sum_{i=1}^{3}\left(\widetilde{\Delta} \boldsymbol{w}_{i}\right) \mathbf{e}_{i}, \\
\widetilde{\boldsymbol{\nabla}} \times \boldsymbol{F} & =\boldsymbol{A} \boldsymbol{\nabla} \times(\boldsymbol{B} \boldsymbol{F}), \\
\widetilde{\nabla} \cdot \boldsymbol{F} & =\frac{1}{\widetilde{d}^{2} d} \nabla \cdot\left(\boldsymbol{A}^{-1} \boldsymbol{F}\right) .
\end{aligned}
$$

Here $\boldsymbol{w}_{i}$ denotes the $i$ 'th component of $\boldsymbol{w}$ and $\mathbf{e}_{i}$ denotes the unit vector in the $i$ 'th direction.

Examining the form of $\nabla \times$ and $\nabla$. in spherical coordinates, one finds that $\widetilde{\nabla} \times \widetilde{\nabla} \times$ and $\widetilde{\nabla} \widetilde{\nabla} \cdot$ involve at most one derivative of $d$ and two derivatives of $\widetilde{d}$, and hence they map smooth functions to continuous ones. They also map functions in $\boldsymbol{H}_{\text {loc }}^{2}\left(\mathbb{R}^{3}\right)$ into $\boldsymbol{L}_{\text {loc }}^{2}\left(\mathbb{R}^{3}\right)$. A analogous argument shows that similar results hold for $\widetilde{\Delta}$ and $\widetilde{\boldsymbol{\Delta}}$.

We shall require three additional identities involving these operators. For completeness, we include their proofs in Appendix A. The first identity is

$$
\widetilde{\Delta} w=-\widetilde{\nabla} \times \widetilde{\nabla} \times w+\widetilde{\nabla} \widetilde{\nabla} \cdot w
$$

for $\boldsymbol{w} \in \boldsymbol{H}_{l o c}^{2}\left(\mathbb{R}^{3}\right)$. The remaining two are the integral identities

$$
\int_{\mathbb{R}^{3}} \widetilde{d}^{2} d(\widetilde{\boldsymbol{\Delta}} \boldsymbol{w}) \cdot(\widetilde{\boldsymbol{\nabla}} \phi) d x=\int_{\mathbb{R}^{3}} \widetilde{d}^{2} d(\widetilde{\boldsymbol{\nabla}}(\widetilde{\nabla} \cdot \boldsymbol{w})) \cdot(\widetilde{\boldsymbol{\nabla}} \phi) d x
$$

and

$$
\int_{\mathbb{R}^{3}} \widetilde{d}^{2} d(\widetilde{\boldsymbol{\Delta}} \boldsymbol{w}) \cdot(\widetilde{\nabla} \times \phi) d x=-\sum_{i=1}^{3} \int_{\mathbb{R}^{3}} \widetilde{d}^{2} d\left(\widetilde{\boldsymbol{\nabla}}(\widetilde{\nabla} \times \boldsymbol{w})_{i}\right) \cdot\left(\widetilde{\nabla} \phi_{i}\right) d x .
$$

These integral identities hold for $\phi \in H^{1}\left(\mathbb{R}^{3}\right), \boldsymbol{\phi} \in \boldsymbol{H}^{1}\left(\mathbb{R}^{3}\right)$ and $\boldsymbol{w} \in \boldsymbol{H}^{2}\left(\mathbb{R}^{3}\right)$.

\section{The elastichave PML Problem}

The "stretched" function $\widetilde{\boldsymbol{u}}$ corresponding to a function $\boldsymbol{u}$ satisfying (2.1) is defined by

$$
\widetilde{\boldsymbol{u}}(\boldsymbol{x})=\left\{\begin{aligned}
\boldsymbol{u}(\boldsymbol{x}): & \text { if } \boldsymbol{x} \in \Omega^{c} \text { and }|\boldsymbol{x}| \leq r_{0}, \\
\sum_{n=0}^{\infty} \sum_{|m| \leq n}\left(\boldsymbol{\alpha}_{n, m} q_{n}(\tilde{r})+\gamma_{n, m} p_{n}(\tilde{r})\right) Y_{n, m}(\hat{\boldsymbol{x}}): & \text { otherwise. }
\end{aligned}\right.
$$

Here $\tilde{r}=(1+i \widetilde{\sigma}(r)) r$, and $\left\{\boldsymbol{\gamma}_{n, m}\right\}$ and $\left\{\boldsymbol{\alpha}_{n, m}\right\}$ are the coefficients appearing in (2.6) and (2.7), respectively. As $p_{n}$ and $q_{n}$ are essentially just Hankel functions, their values for complex arguments are well defined provided one stays away from the branch cut. As the real part of $\tilde{r}$ is greater than zero, $p_{n}$ and $q_{n}$ are well defined if we take the branch cut defining $h_{n}^{(1)}(z)$ to be the negative real axis.

For the proof of our next theorem, we shall require a complex variable argument. To do this, we consider more general PML operators defined by replacing $i$ by $z$ 
with $z \in \widetilde{\mathbb{C}} \equiv\left\{\Re(z)>-1 / \sigma_{M}\right\}$. Here $\sigma_{M}$ denotes the maximum value of $\sigma$. The series defining $\widetilde{\boldsymbol{u}}$ (and all of its derivatives) converges uniformly for $z$ in a compact subset of $\widetilde{\mathbb{C}}$ and for $r$ on compact subsets with $|\tilde{r}|>r_{-1}$.

We have the following theorem.

Theorem 4.1. Let $\widetilde{\boldsymbol{u}}$ be defined by (4.1). Then $\widetilde{\boldsymbol{u}}$ is in $\boldsymbol{H}_{l o c}^{2}\left(\Omega^{c}\right) \cap \boldsymbol{H}^{1}\left(\Omega^{c}\right)$ and satisfies

$$
\widetilde{\boldsymbol{\Delta}} \widetilde{\boldsymbol{u}}+\gamma \widetilde{\boldsymbol{\nabla}} \widetilde{\nabla} \cdot \widetilde{\boldsymbol{u}}+k^{2} \widetilde{\boldsymbol{u}}=\mathbf{0} \quad \text { in } \Omega^{c} .
$$

Proof. Note that $\tilde{r}_{0}=r_{0}$, so (2.8) implies that the transition in (4.1) is smooth. As $\boldsymbol{u} \in \boldsymbol{H}_{l o c}^{2}\left(\Omega^{c}\right), \widetilde{\boldsymbol{u}} \in \boldsymbol{H}_{l o c}^{2}\left(\Omega^{c}\right)$ follows from the uniform convergence of the series in (4.1) and its derivatives.

We consider the complex valued function

$$
F(z)=\int_{\Omega^{c}} \widetilde{d}^{2} d\left(\widetilde{\boldsymbol{\Delta}} \widetilde{\boldsymbol{u}}+\gamma \widetilde{\nabla} \widetilde{\nabla} \cdot \widetilde{\boldsymbol{u}}+k^{2} \widetilde{\boldsymbol{u}}\right) \cdot \boldsymbol{\phi} d x .
$$

Here $\widetilde{\boldsymbol{u}}$ depends on $z$ as $\tilde{r}=(1+z \widetilde{\sigma}) r$ and $\phi \in \boldsymbol{C}_{0}^{\infty}\left(\Omega^{c}\right)$ is real valued and fixed. For real $s$ with $s>-1 / \sigma_{M}$, the transformation $T_{s} \boldsymbol{x}=(1+s \widetilde{\sigma}(r)) \boldsymbol{x}$ maps $\Omega^{c}$ bijectively onto $\Omega^{c}$ and a change of variables gives

$$
F(s)=\left(\boldsymbol{\Delta} \boldsymbol{u}+\gamma \boldsymbol{\nabla} \nabla \cdot \boldsymbol{u}+k^{2} \boldsymbol{u}, \boldsymbol{\phi}_{s}\right)=0 .
$$

Here $\phi_{s}(\boldsymbol{x}) \equiv \phi\left(T_{s}^{-1}(\boldsymbol{x})\right)$. The convergence of the series easily implies that $F$ is analytic on $\widetilde{\mathbb{C}}$. This implies that $F(z)$ vanishes for any $z \in \widetilde{\mathbb{C}}$ from which it immediately follows that $\widetilde{\boldsymbol{\Delta}} \widetilde{\boldsymbol{u}}+\gamma \widetilde{\boldsymbol{\nabla}} \widetilde{\nabla} \cdot \widetilde{\boldsymbol{u}}+k^{2} \widetilde{\boldsymbol{u}}=\mathbf{0}$ for such $z$.

The uniform convergence of the series and its derivatives and Theorem 2.1 imply that $\widetilde{\boldsymbol{u}} \in \boldsymbol{H}^{1}\left(\Omega_{2}\right)$. Outside of $\Omega_{1}$, (4.2) reduces to

$$
\boldsymbol{\Delta} \widetilde{\boldsymbol{u}}+\gamma \nabla \nabla \cdot \widetilde{\boldsymbol{u}}+d_{0}^{2} k^{2} \widetilde{\boldsymbol{u}}=\mathbf{0} .
$$

The corresponding variational problem, find $\boldsymbol{w} \in \boldsymbol{H}^{1}\left(B_{r_{1}}^{c}\right)$ with $\boldsymbol{w}=\widetilde{\boldsymbol{u}}$ on $\Gamma_{1}$ satisfying

$$
\int_{B_{r_{1}}^{c}}\left[\sum_{i=1}^{3} \boldsymbol{\nabla} \boldsymbol{w}_{i} \cdot \boldsymbol{\nabla} \boldsymbol{\Phi}_{i}+\gamma \nabla \cdot \boldsymbol{w} \nabla \cdot \boldsymbol{\Phi}-d_{0}^{2} \boldsymbol{w} \cdot \mathbf{\Phi}\right] d x=0, \quad \text { for all } \boldsymbol{\Phi} \in \boldsymbol{H}_{0}^{1}\left(B_{r_{1}}^{c}\right),
$$

has a unique solution in $\boldsymbol{H}^{1}\left(B_{r_{1}}^{c}\right)$ since $d_{0}^{2}$ has a non-zero imaginary part. As $\boldsymbol{w}$ has a series representation of the form of (4.1) and equals $\widetilde{\boldsymbol{u}}$ on $\Gamma_{1}, \boldsymbol{w}=\widetilde{\boldsymbol{u}}$. This implies that $\widetilde{\boldsymbol{u}} \in \boldsymbol{H}^{1}\left(\Omega^{c}\right)$ and completes the proof.

We revert to the PML shift, i.e., $z=i$, in the remainder of this paper (excluding Appendix A).

For $\boldsymbol{v}, \boldsymbol{\Psi} \in \boldsymbol{H}^{1}\left(\mathbb{R}^{3}\right)$, we define

$$
\begin{aligned}
\mathcal{A}(\boldsymbol{v}, \boldsymbol{\Psi}) & \equiv \sum_{j=1}^{3}\left(\boldsymbol{A}^{-1} \boldsymbol{B}^{-1} \boldsymbol{\nabla} \boldsymbol{v}_{j}, \boldsymbol{\nabla} \boldsymbol{\Psi}_{j}\right)_{\mathbb{R}^{3}} \\
& +\gamma\left(\left(\tilde{d}^{2} d\right)^{-1} \nabla \cdot\left(\boldsymbol{A}^{-1} \boldsymbol{v}\right), \nabla \cdot\left(\overline{\boldsymbol{A}}^{-1} \boldsymbol{\Psi}\right)\right)_{\mathbb{R}^{3}}-k^{2}\left(\widetilde{d}^{2} d \boldsymbol{v}, \boldsymbol{\Psi}\right)_{\mathbb{R}^{3}} .
\end{aligned}
$$

Here $\overline{\boldsymbol{A}}^{-1}$ denotes the complex conjugate. Note that all terms in the above expressions are non-Hermitian.

We shall use the form $\mathcal{A}(\cdot, \cdot)$ to denote the restriction of the above integrals to $D \subseteq \mathbb{R}^{3}$ when one of the two arguments is in $\boldsymbol{H}_{0}^{1}(D)$ and the other is in $\boldsymbol{H}^{1}(D)$. 
Multiplying (4.2) by $\tilde{d}^{2} d \overline{\boldsymbol{\Psi}}$ for $\boldsymbol{\Psi} \in \boldsymbol{C}_{0}^{\infty}\left(\Omega^{c}\right)$, using (3.3), and integrating by parts shows that $\widetilde{\boldsymbol{u}}$ satisfies the weak equations

$$
\mathcal{A}(\widetilde{\boldsymbol{u}}, \boldsymbol{\Psi})=0 .
$$

This equality, of course, extends to all $\boldsymbol{\Psi} \in \boldsymbol{H}_{0}^{1}(D)$ by density.

For the first step in our analysis we consider the source problem on all of $\mathbb{R}^{3}$,

$$
\widetilde{\boldsymbol{\Delta}} \boldsymbol{U}+\gamma \widetilde{\boldsymbol{\nabla}} \widetilde{\nabla} \cdot \boldsymbol{U}+k^{2} \boldsymbol{U}=\boldsymbol{\Phi} \quad \text { in } \mathbb{R}^{3} .
$$

A weak form of this equation is

$$
\mathcal{A}(\boldsymbol{U}, \boldsymbol{\Psi})=\left(\tilde{d}^{2} d \boldsymbol{\Phi}, \boldsymbol{\Psi}\right)_{\mathbb{R}^{3}}, \quad \text { for all } \boldsymbol{\Psi} \in \boldsymbol{H}^{1}\left(\mathbb{R}^{3}\right) .
$$

Let $\widetilde{H}^{1}\left(\mathbb{R}^{3}\right)$ denote the weighted Sobolev space of functions defined on $\mathbb{R}^{3}$ given by

$$
\widetilde{H}^{1}\left(\mathbb{R}^{3}\right)=\left\{u: u\left(1+r^{2}\right)^{-1 / 2} \in L^{2}\left(\mathbb{R}^{3}\right) \text { and } \boldsymbol{\nabla} u \in \boldsymbol{L}^{2}\left(\mathbb{R}^{3}\right)\right\} .
$$

It follows from Theorem 2.5.13 of [12] that $\|\nabla \phi\|_{L^{2}\left(\mathbb{R}^{3}\right)}$ provides an equivalent norm for $\widetilde{H}^{1}\left(\mathbb{R}^{3}\right)$. Moreover, $C_{0}^{\infty}\left(\mathbb{R}^{3}\right)$ is dense in $\widetilde{H}^{1}\left(\mathbb{R}^{3}\right)[12$.

For the analysis of the above problem, we shall need to decompose a vector function $\boldsymbol{\Phi} \in \boldsymbol{L}^{2}\left(\mathbb{R}^{3}\right)$ as

$$
\mathbf{\Phi}=\widetilde{\nabla} \theta+\mathbf{\Phi}_{0},
$$

where $\theta \in \widetilde{H}^{1}\left(\mathbb{R}^{3}\right)$. We choose $\theta$ so that $\widetilde{\nabla} \cdot \mathbf{\Phi}_{0}=0$; specifically, $\theta \in \widetilde{H}^{1}\left(\mathbb{R}^{3}\right)$ is the solution of

$$
\tilde{b}(\theta, \phi)=\left(\boldsymbol{A}^{-1} \mathbf{\Phi}, \boldsymbol{\nabla} \phi\right)_{\mathbb{R}^{3}}, \quad \text { for all } \phi \in \widetilde{H}^{1}\left(\mathbb{R}^{3}\right)
$$

Here

$$
\tilde{b}(\theta, \phi) \equiv\left(\boldsymbol{B}^{-1} \boldsymbol{A}^{-1} \boldsymbol{\nabla} \theta, \boldsymbol{\nabla} \phi\right)_{\mathbb{R}^{3}} .
$$

Since $\boldsymbol{B}^{-1} \boldsymbol{A}^{-1}$ has a uniformly positive definite real part, $\tilde{b}$ is coercive on $\widetilde{H}^{1}\left(\mathbb{R}^{3}\right)$. It follows that (4.7) has a unique solution $\theta \in \widetilde{H}^{1}\left(\mathbb{R}^{3}\right)$ satisfying

$$
\|\nabla \theta\|_{L^{2}\left(\mathbb{R}^{3}\right)} \leq C\|\boldsymbol{\Phi}\|_{\boldsymbol{L}^{2}\left(\mathbb{R}^{3}\right)} .
$$

We shall require the following lemma, whose proof we provide later.

Lemma 4.1. The decomposition (4.6) is stable in $\boldsymbol{H}^{-1}\left(\mathbb{R}^{3}\right)$; i.e.,

$$
\left\|\boldsymbol{\Phi}_{0}\right\|_{\boldsymbol{H}^{-1}\left(\mathbb{R}^{3}\right)}+\|\widetilde{\nabla} \theta\|_{\boldsymbol{H}^{-1}\left(\mathbb{R}^{3}\right)} \leq C\|\boldsymbol{\Phi}\|_{\boldsymbol{H}^{-1}\left(\mathbb{R}^{3}\right)} .
$$

Using the above lemma, we can prove the following theorem.

Theorem 4.2. Let $\boldsymbol{\Phi} \in \boldsymbol{H}^{-1}\left(\mathbb{R}^{3}\right)$. Problem (4.5) has a solution $\boldsymbol{U} \in \boldsymbol{H}^{1}\left(\mathbb{R}^{3}\right)$ satisfying

$$
\|\boldsymbol{U}\|_{H^{1}\left(\mathbb{R}^{3}\right)} \leq C\|\boldsymbol{\Phi}\|_{H^{-1}\left(\mathbb{R}^{3}\right)} .
$$

Proof. We start by analyzing solutions to a scalar PML problem on $\mathbb{R}^{3}$, specifically the solution $u$ of

$$
\widetilde{\Delta} u+k^{2} u=\Phi \text { in } \mathbb{R}^{3}
$$

for $\Phi \in L^{2}\left(\mathbb{R}^{3}\right)$. A weak form of (4.10) is to find $u \in H^{1}\left(\mathbb{R}^{3}\right)$ satisfying

$$
b(u, \theta)=\left(\tilde{d}^{2} \Phi, \theta\right)_{\mathbb{R}^{3}}, \quad \text { for all } \theta \in H^{1}\left(\mathbb{R}^{3}\right) .
$$


Here

$$
b(u, \theta)=\left(\boldsymbol{A}^{-1} \boldsymbol{B}^{-1} \boldsymbol{\nabla} u, \boldsymbol{\nabla}\left(\bar{d}^{-1} \theta\right)\right)_{\mathbb{R}^{3}}-k^{2}\left(\widetilde{d}^{2} u, \theta\right)_{\mathbb{R}^{3}} .
$$

The scattering problem corresponding to (4.10) (for any positive $k$ ) was studied in 2. There it was shown that the form $b(\cdot, \cdot)$ satisfied inf-sup conditions on $H_{0}^{1}\left(\Omega^{c}\right)$. The arguments there extend to the case in which $\Omega^{c}$ is replaced by $\mathbb{R}^{3}$, i.e., for $w \in H^{1}\left(\mathbb{R}^{3}\right)$,

$$
\|u\|_{H^{1}\left(\mathbb{R}^{3}\right)} \leq c \sup _{\phi \in H^{1}\left(\mathbb{R}^{3}\right)} \frac{|b(u, \phi)|}{\|\phi\|_{H^{1}\left(\mathbb{R}^{3}\right)}}
$$

and

$$
\|u\|_{H^{1}\left(\mathbb{R}^{3}\right)} \leq c \sup _{\phi \in H^{1}\left(\mathbb{R}^{3}\right)} \frac{|b(\phi, u)|}{\|\phi\|_{H^{1}\left(\mathbb{R}^{3}\right)}} .
$$

It follows that there is a unique solution $u$ to (4.11) satisfying

$$
\|u\|_{H^{1}\left(\mathbb{R}^{3}\right)} \leq C\|\Phi\|_{H^{-1}\left(\mathbb{R}^{3}\right)} .
$$

Here we use the fact that multiplication by $\widetilde{d}^{2}$ is a bounded operator on $H^{1}\left(\mathbb{R}^{3}\right)$.

The solution $u$ of (4.11), in fact, is in $H^{2}\left(\mathbb{R}^{3}\right)$, and so it solves (4.10). Indeed, since $\widetilde{\sigma} \in C^{2}\left(\mathbb{R}^{+}\right)$and $\sigma \in C^{1}\left(\mathbb{R}^{+}\right)$we see that

$$
\left|b\left(D_{h} v, \phi\right)+b\left(v, D_{-h} \phi\right)\right| \leq C\|v\|_{H^{1}\left(\mathbb{R}^{3}\right)}\|\phi\|_{H^{1}\left(\mathbb{R}^{3}\right)},
$$

where $D_{h}$ is an arbitrary difference quotient of size $h$. Using (4.12) gives

$$
\begin{aligned}
\left\|D_{h} u\right\|_{H^{1}\left(\mathbb{R}^{3}\right)} & \leq C\left(\sup _{\phi \in C_{0}^{\infty}\left(\mathbb{R}^{3}\right)} \frac{\left|b\left(u, D_{-h} \phi\right)\right|}{\|\phi\|_{H^{1}\left(\mathbb{R}^{3}\right)}}+\|u\|_{H^{1}\left(\mathbb{R}^{3}\right)}\right) \\
& =C\left(\sup _{\phi \in C_{0}^{\infty}\left(\mathbb{R}^{3}\right)} \frac{\left|\left(\widetilde{d}^{2} \Phi, D_{-h} \phi\right)\right|}{\|\phi\|_{H^{1}\left(\mathbb{R}^{3}\right)}}+\|u\|_{H^{1}\left(\mathbb{R}^{3}\right)}\right) \\
& \leq C\|\Phi\|_{L^{2}\left(\mathbb{R}^{3}\right)},
\end{aligned}
$$

uniformly in $h$. For the last inequality above, we used (cf. Lemma 8.48 of [17])

$$
\left\|D_{-h} \phi\right\|_{L^{2}\left(\mathbb{R}^{3}\right)} \leq C\|\phi\|_{H^{1}\left(\mathbb{R}^{3}\right)} .
$$

It follows that

$$
\|u\|_{H^{2}\left(\mathbb{R}^{3}\right)} \leq C\|\Phi\|_{L^{2}\left(\mathbb{R}^{3}\right)}
$$

(see, e.g., Lemma 8.49 of [17]).

We next construct a solution of (4.5). We decompose $\boldsymbol{\Phi} \in L^{2}\left(\mathbb{R}^{3}\right)$ as follows:

$$
\boldsymbol{\Phi}=\tilde{\nabla} \theta+(\boldsymbol{\Phi}-\tilde{\nabla} \theta) \equiv \widetilde{\nabla} \theta+\boldsymbol{\Phi}_{0},
$$

where $\theta$ is the solution of (4.7). By construction, $\widetilde{\nabla} \cdot \boldsymbol{\Phi}_{0}=0$.

We then define $\boldsymbol{U}_{1}$ by

$$
\widetilde{\boldsymbol{\Delta}} \boldsymbol{U}_{1}+k^{2} \boldsymbol{U}_{1}=\boldsymbol{\Phi}_{0} .
$$

This equation reduces componentwise to (4.10), with $\Phi$ replaced by $\left(\boldsymbol{\Phi}_{0}\right)_{j}, j=$ $1,2,3$. Thus $\boldsymbol{U}_{1} \in \boldsymbol{H}^{2}\left(\mathbb{R}^{3}\right)$ and

$$
\left\|\boldsymbol{U}_{1}\right\|_{\boldsymbol{H}^{1}\left(\mathbb{R}^{3}\right)} \leq C\left\|\boldsymbol{\Phi}_{0}\right\|_{\boldsymbol{H}^{-1}\left(\mathbb{R}^{3}\right)} .
$$

Multiplying (4.18) by $\widetilde{d}^{2} d \widetilde{\nabla}\left(d^{-1} \bar{\phi}\right)$, and integrating and applying (3.5) gives us that $\widetilde{\nabla} \cdot \boldsymbol{U}_{1}$ satisfies

$$
b\left(\widetilde{\nabla} \cdot U_{1}, \phi\right)=0 \quad \text { for all } \phi \in H^{1}\left(\mathbb{R}^{3}\right) .
$$


It follows from (4.12) that $\widetilde{\nabla} \cdot \boldsymbol{U}_{1}=0$, so $\boldsymbol{U}_{1}$ satisfies

$$
\widetilde{\boldsymbol{\Delta}} \boldsymbol{U}_{1}+\gamma \widetilde{\boldsymbol{\nabla}}\left(\widetilde{\nabla} \cdot \boldsymbol{U}_{1}\right)+k^{2} \boldsymbol{U}_{1}=\boldsymbol{\Phi}_{0} \text { in } \mathbb{R}^{3}
$$

Next define $\boldsymbol{U}_{2}$ to be the solution of

$$
(1+\gamma) \widetilde{\Delta} U_{2}+k^{2} \boldsymbol{U}_{2}=\widetilde{\nabla} \theta
$$

This equation also reduces componentwise to (4.10), with $k^{2}$ replaced by $k^{2}(1+\gamma)^{-1}$ and $\Phi$ replaced by $(1+\gamma)^{-1}(\widetilde{\nabla} \theta)_{i}, i=1,2,3$. Thus $\boldsymbol{U}_{2} \in \boldsymbol{H}^{2}\left(\mathbb{R}^{3}\right)$ and

$$
\left\|\boldsymbol{U}_{2}\right\|_{\boldsymbol{H}^{1}\left(\mathbb{R}^{3}\right)} \leq C\|\widetilde{\nabla} \theta\|_{\boldsymbol{H}^{-1}\left(\mathbb{R}^{3}\right)} .
$$

Multiplying (4.21) by $\widetilde{d}^{2} d \widetilde{\nabla} \times\left(d^{-1} \overline{\boldsymbol{\phi}}\right)$, and integrating and applying (3.6) shows that each component of $\widetilde{\nabla} \times U_{2}$ satisfies

$$
b\left(\left(\widetilde{\nabla} \times \boldsymbol{U}_{2}\right)_{j}, \phi\right)=0 \quad \text { for all } \phi \in H^{1}\left(\mathbb{R}^{3}\right),
$$

with $b$ defined using $k_{1}$. As above, this implies that $\widetilde{\nabla} \times \boldsymbol{U}_{2}=\mathbf{0}$. It follows from (4.21) and (3.4) that $\boldsymbol{U}_{2}$ satisfies

$$
\widetilde{\boldsymbol{\Delta}} \boldsymbol{U}_{2}+\gamma \widetilde{\boldsymbol{\nabla}}\left(\widetilde{\nabla} \cdot \boldsymbol{U}_{2}\right)+k^{2} \boldsymbol{U}_{2}=\tilde{\nabla} \theta \text { in } \mathbb{R}^{3}
$$

Finally, we define $\boldsymbol{U}=\boldsymbol{U}_{1}+\boldsymbol{U}_{2}$. Clearly, $\boldsymbol{U}$ satisfies (4.4) and (4.9) follows from (4.19), (4.22) and Lemma 4.1. This completes the proof of the theorem.

Proof of Lemma 4.1. It suffices to show that

$$
\left\|\boldsymbol{\Phi}_{0}\right\|_{\boldsymbol{H}^{-1}\left(\mathbb{R}^{3}\right)} \leq C\|\boldsymbol{\Phi}\|_{\boldsymbol{H}^{-1}\left(\mathbb{R}^{3}\right)} .
$$

Given $\boldsymbol{\Psi} \in \boldsymbol{H}^{1}\left(\mathbb{R}^{3}\right)$, let $\eta \in \widetilde{H}^{1}\left(\mathbb{R}^{3}\right)$ solve the (adjoint) problem

$$
\tilde{b}(\delta, \eta)=(\widetilde{\nabla} \delta, \Psi)_{\mathbb{R}^{3}}, \quad \text { for all } \delta \in \widetilde{H}^{1}\left(\mathbb{R}^{3}\right) .
$$

Clearly,

$$
\|\nabla \eta\|_{L^{2}\left(\mathbb{R}^{3}\right)} \leq C\|\boldsymbol{\Psi}\|_{\boldsymbol{L}^{2}\left(\mathbb{R}^{3}\right)} .
$$

Moreover, for any first difference quotient $D_{h}$ of size $h<1$, it follows easily from integration by parts and (4.24) that

$$
\left|\tilde{b}\left(\delta, D_{h} \eta\right)\right| \leq C\|\boldsymbol{\nabla} \delta\|_{\boldsymbol{L}^{2}\left(\mathbb{R}^{3}\right)}\|\boldsymbol{\Psi}\|_{\boldsymbol{H}^{1}\left(\mathbb{R}^{3}\right)},
$$

with $C$ independent of $h$. From this it follows that

$$
\|\nabla \eta\|_{\boldsymbol{H}^{1}\left(\mathbb{R}^{3}\right)} \leq C\|\boldsymbol{\Psi}\|_{\boldsymbol{H}^{1}\left(\mathbb{R}^{3}\right)} .
$$

We then have

$$
\begin{aligned}
\left\|\boldsymbol{\Phi}_{0}\right\|_{\boldsymbol{H}^{-1}\left(\mathbb{R}^{3}\right)} & =\sup _{\boldsymbol{\Psi} \in \boldsymbol{H}^{1}\left(\mathbb{R}^{3}\right)} \frac{\left|\left(\boldsymbol{\Phi}_{0}, \boldsymbol{\Psi}\right)_{\mathbb{R}^{3}}\right|}{\|\boldsymbol{\Psi}\|_{\boldsymbol{H}^{1}\left(\mathbb{R}^{3}\right)}}=\sup _{\boldsymbol{\Psi} \in \boldsymbol{H}^{1}\left(\mathbb{R}^{3}\right)} \frac{\left|\left(\boldsymbol{\Phi}_{0}, \boldsymbol{\Psi}-\bar{A}^{-1} \boldsymbol{\nabla} \eta\right)_{\mathbb{R}^{3}}\right|}{\|\boldsymbol{\Psi}\|_{\boldsymbol{H}^{1}\left(\mathbb{R}^{3}\right)}} \\
& =\sup _{\boldsymbol{\Psi} \in \boldsymbol{H}^{1}\left(\mathbb{R}^{3}\right)} \frac{\left|\left(\boldsymbol{\Phi}, \boldsymbol{\Psi}-\bar{A}^{-1} \boldsymbol{\nabla} \eta\right)_{\mathbb{R}^{3}}\right|}{\|\boldsymbol{\Psi}\|_{\boldsymbol{H}^{1}\left(\mathbb{R}^{3}\right)}} .
\end{aligned}
$$

Combining this with (4.25) gives (4.23) and completes the proof of the lemma.

We have constructed a solution of (4.5) which satisfies (4.9). We next show that this is the unique solution of (4.5).

Corollary 4.1. Let $\boldsymbol{W} \in \boldsymbol{H}^{1}\left(\mathbb{R}^{3}\right)$ satisfy $\mathcal{A}(\boldsymbol{W}, \boldsymbol{\Theta})=0$ for all $\boldsymbol{\Theta} \in \boldsymbol{H}^{1}\left(\mathbb{R}^{3}\right)$. Then $\boldsymbol{W}=\mathbf{0}$. 
Proof. Let $\boldsymbol{W}$ be as above. By Theorem 4.2, there is a solution $\boldsymbol{x}$ of

$$
\mathcal{A}(\boldsymbol{x}, \boldsymbol{\Theta})=(\overline{\boldsymbol{W}}, \boldsymbol{\Theta})_{\mathbb{R}^{3}} \quad \text { for all } \boldsymbol{\Theta} \in \boldsymbol{H}^{1}\left(\mathbb{R}^{3}\right) .
$$

Thus

$$
\|\overline{\boldsymbol{W}}\|_{\boldsymbol{L}^{2}\left(\mathbb{R}^{3}\right)}^{2}=\mathcal{A}(\boldsymbol{W}, \overline{\boldsymbol{x}})=0 .
$$

Hence $\boldsymbol{W}=\mathbf{0}$.

The above corollary implies the inf-sup conditions of the following theorem.

Theorem 4.3. Let $\boldsymbol{U}$ be in $\boldsymbol{H}^{1}\left(\mathbb{R}^{3}\right)$. Then

$$
\|\boldsymbol{U}\|_{\boldsymbol{H}^{1}\left(\mathbb{R}^{3}\right)} \leq C \sup _{\boldsymbol{V} \in \boldsymbol{H}^{1}\left(\mathbb{R}^{3}\right)} \frac{|\mathcal{A}(\boldsymbol{U}, \boldsymbol{V})|}{\|\boldsymbol{V}\|_{\boldsymbol{H}^{1}\left(\mathbb{R}^{3}\right)}}
$$

and

$$
\|\boldsymbol{U}\|_{\boldsymbol{H}^{1}\left(\mathbb{R}^{3}\right)} \leq C \sup _{\boldsymbol{V} \in \boldsymbol{H}^{1}\left(\mathbb{R}^{3}\right)} \frac{|\mathcal{A}(\boldsymbol{V}, \boldsymbol{U})|}{\|\boldsymbol{V}\|_{\boldsymbol{H}^{1}\left(\mathbb{R}^{3}\right)}}
$$

Proof. Let $\boldsymbol{U}$ be in $\boldsymbol{H}^{1}\left(\mathbb{R}^{3}\right)$. Multiplication by a uniformly bounded bijective $C^{1}$ function whose inverse is also uniformly bounded is an isomorphism of $H^{1}\left(\mathbb{R}^{3}\right)$ onto $H^{1}\left(\mathbb{R}^{3}\right)$ and hence is also an isomorphism of $H^{-1}\left(\mathbb{R}^{3}\right)$ onto $H^{-1}\left(\mathbb{R}^{3}\right)$. Thus, $\mathcal{A}(\boldsymbol{U}, \cdot)$ defines an element $\boldsymbol{\Phi}$ of $\boldsymbol{H}^{-1}\left(\mathbb{R}^{3}\right)$ by

$$
\left\langle\widetilde{d}^{2} d \boldsymbol{\Phi}, \boldsymbol{V}\right\rangle=\mathcal{A}(\boldsymbol{U}, \boldsymbol{V}) .
$$

Here $\langle\cdot, \cdot\rangle$ denotes the duality pairing. The above corollary shows that $\boldsymbol{U}$ coincides with the function constructed in Theorem 4.2. Thus,

$$
\begin{aligned}
\|\boldsymbol{U}\|_{\boldsymbol{H}^{1}\left(\mathbb{R}^{3}\right)} & \leq C\|\boldsymbol{\Phi}\|_{\boldsymbol{H}^{-1}\left(\mathbb{R}^{3}\right)} \leq C\left\|\widetilde{d}^{2} d \boldsymbol{\Phi}\right\|_{\boldsymbol{H}^{-1}\left(\mathbb{R}^{3}\right)} \\
& =C \sup _{\boldsymbol{V} \in \boldsymbol{H}^{1}\left(\mathbb{R}^{3}\right)} \frac{|\mathcal{A}(\boldsymbol{U}, \boldsymbol{V})|}{\|\boldsymbol{V}\|_{\boldsymbol{H}^{1}\left(\mathbb{R}^{3}\right)}}
\end{aligned}
$$

This is the first inf-sup condition.

The second inf-sup condition follows from the first; indeed,

$$
\|\boldsymbol{U}\|_{\boldsymbol{H}^{1}\left(\mathbb{R}^{3}\right)}=\|\overline{\boldsymbol{U}}\|_{\boldsymbol{H}^{1}\left(\mathbb{R}^{3}\right)} \leq C \sup _{\boldsymbol{V} \in \boldsymbol{H}^{1}\left(\mathbb{R}^{3}\right)} \frac{|\mathcal{A}(\overline{\boldsymbol{U}}, \boldsymbol{V})|}{\|\boldsymbol{V}\|_{\boldsymbol{H}^{1}\left(\mathbb{R}^{3}\right)}}=\sup _{\boldsymbol{V} \in \boldsymbol{H}^{1}\left(\mathbb{R}^{3}\right)} \frac{|\mathcal{A}(\boldsymbol{V}, \boldsymbol{U})|}{\|\boldsymbol{V}\|_{\boldsymbol{H}^{1}\left(\mathbb{R}^{3}\right)}} .
$$

We next consider the form (4.3) restricted to $\boldsymbol{H}_{0}^{1}\left(\Omega^{c}\right)$ and prove the analogous theorem.

Theorem 4.4. Let $\boldsymbol{U}$ be in $\boldsymbol{H}_{0}^{1}\left(\Omega^{c}\right)$. Then

$$
\|\boldsymbol{U}\|_{\boldsymbol{H}^{1}\left(\Omega^{c}\right)} \leq C \sup _{\boldsymbol{V} \in \boldsymbol{H}_{0}^{1}\left(\Omega^{c}\right)} \frac{|\mathcal{A}(\boldsymbol{U}, \boldsymbol{V})|}{\|\boldsymbol{V}\|_{\boldsymbol{H}^{1}\left(\Omega^{c}\right)}}
$$

and

$$
\|\boldsymbol{U}\|_{\boldsymbol{H}^{1}\left(\Omega^{c}\right)} \leq C \sup _{\boldsymbol{V} \in \boldsymbol{H}_{0}^{1}\left(\Omega^{c}\right)} \frac{|\mathcal{A}(\boldsymbol{V}, \boldsymbol{U})|}{\|\boldsymbol{V}\|_{\boldsymbol{H}^{1}\left(\Omega^{c}\right)}} .
$$


Proof. Let $\boldsymbol{\Phi} \in \boldsymbol{H}^{-1}\left(\Omega^{c}\right)$. By the Hahn-Banach Theorem we may extend $\boldsymbol{\Phi}$ to $\boldsymbol{H}^{-1}\left(\mathbb{R}^{3}\right)$ with

$$
\|\Phi\|_{H^{-1}\left(\mathbb{R}^{3}\right)}=\|\Phi\|_{H^{-1}\left(\Omega^{c}\right)} .
$$

By Theorem 4.2, there is a unique solution $\boldsymbol{W}$ of

$$
\mathcal{A}(\boldsymbol{W}, \boldsymbol{\Theta})=\langle\boldsymbol{\Phi}, \boldsymbol{\Theta}\rangle, \quad \text { for all } \boldsymbol{\Theta} \in \boldsymbol{H}^{1}\left(\mathbb{R}^{3}\right) .
$$

Now let $\boldsymbol{U}_{0}$ be the solution of the elastic wave problem satisfying (2.1), (2.4), (2.5) and (2.2) with $\boldsymbol{g}=\boldsymbol{W}$ on $\Gamma$. Also let $\widetilde{\boldsymbol{U}_{0}}$ be the solution of the corresponding PML elastic wave problem given by (4.1) (with $\boldsymbol{u}$ replaced by $\boldsymbol{U}_{0}$ ). By (2.9),

$$
\left\|\widetilde{\boldsymbol{U}_{0}}\right\|_{\boldsymbol{H}^{1}\left(\Omega_{0}\right)}=\left\|\boldsymbol{U}_{0}\right\|_{\boldsymbol{H}^{1}\left(\Omega_{0}\right)} \leq C\|\boldsymbol{W}\|_{\boldsymbol{H}^{1 / 2}(\Gamma)} .
$$

We will show that

$$
\left\|\widetilde{\boldsymbol{U}_{0}}\right\|_{\boldsymbol{H}^{1}\left(\Omega^{c}\right)} \leq C\|\boldsymbol{W}\|_{\boldsymbol{H}^{1 / 2}(\Gamma)} .
$$

Let $\psi$ be a smooth cut-off function satisfying $\psi(\boldsymbol{x})=1$ for $|\boldsymbol{x}| \geq r_{0}$ and $\psi(\boldsymbol{x})=0$ on $\Gamma$. Let $\boldsymbol{V} \equiv \psi \widetilde{\boldsymbol{U}}_{0}$ in $\Omega^{c}$ be extended by $\mathbf{0}$ to all of $\mathbb{R}^{3}$. Let $\boldsymbol{\phi}$ be in $\boldsymbol{H}^{1}\left(\mathbb{R}^{3}\right)$. Since

it follows that

$$
\mathcal{A}\left(\widetilde{\boldsymbol{U}_{0}}, \boldsymbol{\Theta}\right)=0, \quad \text { for all } \boldsymbol{\Theta} \in \boldsymbol{H}_{0}^{1}\left(\Omega^{c}\right),
$$

$$
|\mathcal{A}(\boldsymbol{V}, \boldsymbol{\phi})|=\left|\mathcal{A}\left((\psi-1) \widetilde{\boldsymbol{U}}_{0}, \boldsymbol{\phi}\right)+\mathcal{A}\left(\widetilde{\boldsymbol{U}}_{0},(1-\psi) \phi\right)\right| \leq C\left\|\widetilde{\boldsymbol{U}}_{0}\right\|_{\boldsymbol{H}^{1}\left(\Omega_{0}\right)}\|\boldsymbol{\phi}\|_{\boldsymbol{H}^{1}\left(\mathbb{R}^{3}\right)} .
$$

Applying (4.26) gives

We then have

$$
\|\boldsymbol{V}\|_{\boldsymbol{H}^{1}\left(\mathbb{R}^{3}\right)} \leq C\left\|\widetilde{\boldsymbol{U}}_{0}\right\|_{\boldsymbol{H}^{1}\left(\Omega_{0}\right)} .
$$

$$
\left\|\widetilde{\boldsymbol{U}}_{0}\right\|_{\boldsymbol{H}^{1}\left(\Omega^{c}\right)} \leq\|\boldsymbol{V}\|_{\boldsymbol{H}^{1}\left(\Omega^{c}\right)}+\left\|(1-\psi) \widetilde{\boldsymbol{U}}_{0}\right\|_{\boldsymbol{H}^{1}\left(\Omega_{0}\right)} \leq C\left\|\widetilde{\boldsymbol{U}}_{0}\right\|_{\boldsymbol{H}^{1}\left(\Omega_{0}\right)},
$$

and (4.31) follows from (4.30).

Now set $\boldsymbol{U}=\boldsymbol{W}-\widetilde{\boldsymbol{U}_{0}}$. We have constructed a vector function $\boldsymbol{U} \in \boldsymbol{H}^{1}\left(\Omega^{c}\right)$ such that

for any $\boldsymbol{\Phi} \in \boldsymbol{H}^{-1}\left(\Omega^{c}\right)$. Also,

$$
\mathcal{A}(\boldsymbol{U}, \boldsymbol{\Theta})=\langle\boldsymbol{\Phi}, \boldsymbol{\Theta}\rangle, \quad \text { for all } \boldsymbol{\Theta} \in \boldsymbol{H}_{0}^{1}\left(\Omega^{c}\right),
$$

$$
\begin{aligned}
\|\boldsymbol{U}\|_{\boldsymbol{H}^{1}\left(\Omega^{c}\right)} & \leq\|\boldsymbol{W}\|_{\boldsymbol{H}^{1}\left(\Omega^{c}\right)}+\left\|\widetilde{\boldsymbol{U}_{0}}\right\|_{\boldsymbol{H}^{1}\left(\Omega^{c}\right)} \\
& \leq C\left(\|\boldsymbol{W}\|_{\boldsymbol{H}^{1}\left(\Omega^{c}\right)}+\|\boldsymbol{W}\|_{\boldsymbol{H}^{1 / 2}(\Gamma)}\right) \leq C\|\boldsymbol{\Phi}\|_{\boldsymbol{H}^{-1}\left(\Omega^{c}\right)} .
\end{aligned}
$$

The arguments proving Theorems 4.1 and 4.3 now complete the proof of the theorem.

\section{The truncated PML Problem}

In this section, we consider approximating the PML problem on $\Omega^{c}$ by a problem on a truncated domain with a convenient boundary condition on the outer boundary. For convenience, we shall use homogeneous Dirichlet conditions. Let $\Omega_{\infty}$ be a bounded subset of $\Omega^{c}$ containing the transition region, i.e., $\Omega_{1} \subseteq \Omega_{\infty}$. The size of $\Omega_{\infty}$ is controlled by a parameter $R_{t}$; for example, the outer boundary $\Gamma_{\infty}$ of $\Omega_{\infty}$ could be a cube with edge of length $2 R_{t}$. In any event, we assume that $\Gamma_{\infty}$ is uniformly Lipschitz with constants that are independent of $R_{t}$ and that $B_{R_{t}} \subseteq \Omega_{\infty}$.

We are interested in studying the truncated variational problem. Find $\boldsymbol{U} \in$ $\boldsymbol{H}_{0}^{1}\left(\Omega_{\infty}\right)$ satisfying

$$
\mathcal{A}(\boldsymbol{U}, \phi)=\langle\boldsymbol{F}, \phi\rangle, \quad \text { for all } \boldsymbol{\phi} \in \boldsymbol{H}_{0}^{1}\left(\Omega_{\infty}\right)
$$


One of our main tasks will be to show that this problem is well posed for $R_{t}$ sufficiently large. This is a consequence of the following theorem.

Theorem 5.1. Let $\boldsymbol{U}$ be in $\boldsymbol{H}_{0}^{1}\left(\Omega_{\infty}\right)$. Then, for $R_{t}$ sufficiently large,

$$
\|\boldsymbol{U}\|_{\boldsymbol{H}^{1}\left(\Omega_{\infty}\right)} \leq C \sup _{\boldsymbol{V} \in \boldsymbol{H}_{0}^{1}\left(\Omega_{\infty}\right)} \frac{|\mathcal{A}(\boldsymbol{U}, \boldsymbol{V})|}{\|\boldsymbol{V}\|_{\boldsymbol{H}^{1}\left(\Omega_{\infty}\right)}}
$$

and

$$
\|\boldsymbol{U}\|_{\boldsymbol{H}^{1}\left(\Omega_{\infty}\right)} \leq C \sup _{\boldsymbol{V} \in \boldsymbol{H}_{0}^{1}\left(\Omega_{\infty}\right)} \frac{|\mathcal{A}(\boldsymbol{V}, \boldsymbol{U})|}{\|\boldsymbol{V}\|_{\boldsymbol{H}^{1}\left(\Omega_{\infty}\right)}} .
$$

We will need the following two lemmas. These lemmas will be used extensively throughout the remainder of this paper. The first is a standard application of the Lax-Milgram Lemma. The second is a decay estimate.

Lemma 5.1. Let $D$ be a domain $\mathbb{R}^{3}$ with a Lipschitz boundary $\partial D$ and let $\mathcal{B}$ be a bounded sesquilinear form on $\boldsymbol{H}^{1}(D) \times \boldsymbol{H}^{1}(D)$ satisfying, for $\boldsymbol{u} \in \boldsymbol{H}_{0}^{1}(D)$,

$$
\|\boldsymbol{u}\|_{\boldsymbol{H}^{1}(D)} \leq C \sup _{\boldsymbol{\phi} \in \boldsymbol{H}_{0}^{1}(D)} \frac{|\mathcal{B}(\boldsymbol{u}, \boldsymbol{\phi})|}{\|\boldsymbol{\phi}\|_{\boldsymbol{H}^{1}(D)}}
$$

and

$$
\|\boldsymbol{u}\|_{\boldsymbol{H}^{1}(D)} \leq C \sup _{\boldsymbol{\phi} \in \boldsymbol{H}_{0}^{1}(D)} \frac{|\mathcal{B}(\boldsymbol{\phi}, \boldsymbol{u})|}{\|\boldsymbol{\phi}\|_{\boldsymbol{H}^{1}(D)}} .
$$

Then given $\boldsymbol{v} \in \boldsymbol{H}^{1 / 2}(\partial D)$ there exists a unique $\boldsymbol{u} \in \boldsymbol{H}^{1}(D)$ with $\boldsymbol{u}=\boldsymbol{v}$ on $\partial D$ satisfying

$$
\mathcal{B}(\boldsymbol{u}, \boldsymbol{\phi})=0, \quad \text { for all } \boldsymbol{\phi} \in \boldsymbol{H}_{0}^{1}(D) .
$$

Furthermore,

$$
\|\boldsymbol{u}\|_{\boldsymbol{H}^{1}(D)} \leq C\|\boldsymbol{v}\|_{\boldsymbol{H}^{1 / 2}(\partial D)} .
$$

The next lemma is a decay estimate associated with elastic wave equation. We will include its proof in Appendix B.

Lemma 5.2. Assume that $\boldsymbol{u} \in \boldsymbol{H}^{1}\left(\Omega_{1}^{c}\right)$ and satisfies

$$
\boldsymbol{\Delta} \boldsymbol{u}+\gamma \boldsymbol{\nabla} \nabla \cdot \boldsymbol{u}+k^{2} d_{0}^{2} \boldsymbol{u}=\mathbf{0}
$$

in $\Omega_{1}^{c}$. Then,

$$
\|\boldsymbol{u}\|_{\boldsymbol{H}^{1 / 2}\left(\Gamma_{\infty}\right)} \leq C e^{-\sigma_{0} k_{1} R_{t}}\|\boldsymbol{u}\|_{\boldsymbol{H}^{1}\left(\Omega_{\infty}\right)} .
$$

Proof of Theorem [5.1. We will prove (5.1). Inequality (5.2) follows from (5.1) as in the proof of (4.27). For $\boldsymbol{U} \in \boldsymbol{H}_{0}^{1}\left(\Omega_{\infty}\right)$, we apply (4.28) to conclude

$$
\|\boldsymbol{U}\|_{\boldsymbol{H}_{0}^{1}\left(\Omega_{\infty}\right)} \leq C \sup _{\boldsymbol{V} \in \boldsymbol{H}_{0}^{1}\left(\Omega^{c}\right)} \frac{\left|\mathcal{A}\left(\boldsymbol{U}, \boldsymbol{V}_{0}\right)+\mathcal{A}\left(\boldsymbol{U}, \boldsymbol{V}_{1}\right)\right|}{\|\boldsymbol{V}\|_{\boldsymbol{H}^{1}\left(\Omega^{c}\right)}},
$$

where we have decomposed $\boldsymbol{V}=\boldsymbol{V}_{0}+\boldsymbol{V}_{1}$ with $\boldsymbol{V}_{1}$ satisfying $\boldsymbol{V}_{1}=\boldsymbol{V}$ on $\Omega_{\infty}^{c}$, $\boldsymbol{V}_{1}=\mathbf{0}$ on $\Omega_{1}$ and

$$
\mathcal{A}\left(\phi, \boldsymbol{V}_{1}\right)=0, \quad \text { for all } \phi \in \boldsymbol{H}_{0}^{1}\left(\Omega_{\infty} \backslash \bar{\Omega}_{1}\right) .
$$


The above problem is well posed. Indeed, for $\phi \in \boldsymbol{H}_{0}^{1}(D)$, where $D$ is any subdomain of $\Omega_{1}^{c}$,

$$
\begin{aligned}
|\mathcal{A}(\boldsymbol{\phi}, \boldsymbol{\phi})| & =\left|d_{0}\right|^{2}\left|\mathcal{A}\left(\boldsymbol{\phi}, \bar{d}_{0}^{-2} \boldsymbol{\phi}\right)\right| \\
& \geq-\left|d_{0}\right|^{2} \operatorname{Im}\left(\mathcal{A}\left(\boldsymbol{\phi}, \bar{d}_{0}^{-2} \phi\right)\right) \geq \sigma_{0} \min \left(1, k^{2}\right)\|\boldsymbol{\phi}\|_{\boldsymbol{H}^{1}(D)}^{2}
\end{aligned}
$$

It follows from Lemma 5.1 that $\boldsymbol{V}_{1}$ is unique and satisfies

$$
\left\|\boldsymbol{V}_{1}\right\|_{\boldsymbol{H}^{1}\left(\Omega_{\infty} \backslash \bar{\Omega}_{1}\right)} \leq C\|\boldsymbol{V}\|_{\boldsymbol{H}^{1 / 2}\left(\Gamma_{\infty}\right)} \leq C\|\boldsymbol{V}\|_{\boldsymbol{H}^{1}\left(\Omega_{\infty} \backslash \bar{\Omega}_{1}\right)} .
$$

Let $\widetilde{\boldsymbol{U}}$ solve $\widetilde{\boldsymbol{U}}=\boldsymbol{U}$ on $\Omega_{1}$ and

$$
\mathcal{A}(\widetilde{\boldsymbol{U}}, \boldsymbol{\phi})=0, \quad \text { for all } \boldsymbol{\phi} \in \boldsymbol{H}_{0}^{1}\left(\Omega_{1}^{c}\right)
$$

As above, from Lemma 5.1, $\widetilde{\boldsymbol{U}}$ is unique in $\boldsymbol{H}^{1}\left(\Omega_{1}^{c}\right)$ and satisfies

$$
\|\widetilde{\boldsymbol{U}}\|_{\boldsymbol{H}^{1}\left(\Omega_{1}^{c}\right)} \leq C\|\boldsymbol{U}\|_{\boldsymbol{H}^{1 / 2}\left(\Gamma_{1}\right)} \leq C\|\boldsymbol{U}\|_{\boldsymbol{H}^{1}\left(\Omega_{\infty}\right)} .
$$

We also define $\boldsymbol{U}_{1}$ by $\boldsymbol{U}_{1}=\boldsymbol{U}$ on $\Gamma_{1}, \boldsymbol{U}_{1}=\mathbf{0}$ on $\Omega_{\infty}^{c}$, and

$$
\mathcal{A}\left(\boldsymbol{U}_{1}, \boldsymbol{\phi}\right)=0, \quad \text { for all } \boldsymbol{\phi} \in \boldsymbol{H}_{0}^{1}\left(\Omega_{\infty} \backslash \bar{\Omega}_{1}\right) .
$$

Again, from Lemma 5.1, $\boldsymbol{U}_{1}$ is unique in $\boldsymbol{H}^{1}\left(\Omega_{\infty} \backslash \bar{\Omega}_{1}\right)$, and we have that

$$
\mathcal{A}\left(\boldsymbol{U}_{1}-\widetilde{\boldsymbol{U}}, \boldsymbol{\phi}\right)=0, \quad \text { for all } \boldsymbol{\phi} \in \boldsymbol{H}_{0}^{1}\left(\Omega_{\infty} \backslash \bar{\Omega}_{1}\right) .
$$

Since $\boldsymbol{V}_{1} \in \boldsymbol{H}_{0}^{1}\left(\Omega_{1}^{c}\right)$ and $\boldsymbol{U}-\boldsymbol{U}_{1} \in \boldsymbol{H}_{0}^{1}\left(\Omega_{\infty} \backslash \bar{\Omega}_{1}\right)$, (5.8) and (5.6) imply

$$
\begin{aligned}
\left|\mathcal{A}\left(\boldsymbol{U}, \boldsymbol{V}_{1}\right)\right| & =\left|\mathcal{A}\left(\boldsymbol{U}_{1}-\tilde{\boldsymbol{U}}, \boldsymbol{V}_{1}\right)\right| \\
& \leq C\left\|\boldsymbol{U}_{1}-\widetilde{\boldsymbol{U}}\right\|_{\boldsymbol{H}^{1}\left(\Omega_{1}^{c}\right)}\left\|\boldsymbol{V}_{1}\right\|_{\boldsymbol{H}^{1}\left(\Omega_{\infty} \backslash \bar{\Omega}_{1}\right)} .
\end{aligned}
$$

Because of (5.10) and Lemma 5.1

$$
\left\|\boldsymbol{U}_{1}-\widetilde{\boldsymbol{U}}\right\|_{\boldsymbol{H}^{1}\left(\Omega_{\infty} \backslash \bar{\Omega}_{1}\right)} \leq C\|\widetilde{\boldsymbol{U}}\|_{\boldsymbol{H}^{1 / 2}\left(\Gamma_{\infty}\right)} .
$$

Also because of (5.9) and Lemma [5.1.

$$
\left\|\boldsymbol{U}_{1}-\widetilde{\boldsymbol{U}}\right\|_{\boldsymbol{H}^{1}\left(\Omega_{\infty}^{c}\right)}=\|\widetilde{\boldsymbol{U}}\|_{\boldsymbol{H}^{1}\left(\Omega_{\infty}^{c}\right)} \leq C\|\widetilde{\boldsymbol{U}}\|_{\boldsymbol{H}^{1 / 2}\left(\Gamma_{\infty}\right)} .
$$

Combining the last three inequalities, (5.7) and Lemma 5.2 gives

$$
\left|\mathcal{A}\left(\boldsymbol{U}, \boldsymbol{V}_{1}\right)\right| \leq C\|\widetilde{\boldsymbol{U}}\|_{\boldsymbol{H}^{1 / 2}\left(\Gamma_{\infty}\right)}\|\boldsymbol{V}\|_{\boldsymbol{H}^{1}\left(\Omega_{\infty}\right)} \leq C e^{-\sigma_{0} k_{1} R_{t}}\|\boldsymbol{U}\|_{\boldsymbol{H}^{1}\left(\Omega_{\infty}\right)}\|\boldsymbol{V}\|_{\boldsymbol{H}^{1}\left(\Omega_{\infty}\right)} .
$$

Thus,

$$
\|\boldsymbol{U}\|_{H^{1}\left(\Omega_{\infty}\right)} \leq C \sup _{\boldsymbol{V}_{0} \in H_{0}^{1}\left(\Omega_{\infty}\right)} \frac{\left|\mathcal{A}\left(\boldsymbol{U}, \boldsymbol{V}_{0}\right)\right|}{\left\|\boldsymbol{V}_{0}\right\|_{H^{1}\left(\Omega_{\infty}\right)}}+C e^{-\sigma_{0} k_{1} R_{t}}\|\boldsymbol{U}\|_{\boldsymbol{H}^{1}\left(\Omega_{\infty}\right)} .
$$

Inequality (5.1) follows by taking $R_{t}$ sufficiently large. This completes the proof of the theorem.

We finally prove that the truncated elasticwave PML solution $\widetilde{\boldsymbol{u}}_{t}$ converges exponentially to the elasticwave solution in $\boldsymbol{H}^{1}\left(\Omega_{0}\right)$.

Theorem 5.2. Assume that $R_{t}$ is large enough that Theorem 5.1 holds. Let $\widetilde{\boldsymbol{u}}$ be in $\boldsymbol{H}^{1}\left(\Omega^{c}\right)$ and satisfy

$$
\mathcal{A}(\widetilde{\boldsymbol{u}}, \boldsymbol{\phi})=0, \quad \text { for all } \boldsymbol{\phi} \in \boldsymbol{H}_{0}^{1}\left(\Omega^{c}\right) .
$$

Let $\widetilde{\boldsymbol{u}}_{t}$ be in $\boldsymbol{H}^{1}\left(\Omega_{\infty}\right)$ and satisfy

$$
\mathcal{A}\left(\widetilde{\boldsymbol{u}}_{t}, \boldsymbol{\phi}\right)=0, \quad \text { for all } \boldsymbol{\phi} \in \boldsymbol{H}_{0}^{1}\left(\Omega_{\infty}\right)
$$


and $\widetilde{\boldsymbol{u}}_{t}=\boldsymbol{u}$ on $\Gamma$ and $\widetilde{\boldsymbol{u}}_{t}=\mathbf{0}$ on $\Gamma_{\infty}$. Then

$$
\left\|\widetilde{\boldsymbol{u}}-\widetilde{\boldsymbol{u}}_{t}\right\|_{\boldsymbol{H}^{1}\left(\Omega_{\infty}\right)} \leq C e^{-\sigma_{0} k_{1} R_{t}}\|\boldsymbol{u}\|_{\boldsymbol{H}^{1 / 2}(\Gamma)}
$$

and hence

$$
\left\|\boldsymbol{u}-\widetilde{\boldsymbol{u}}_{t}\right\|_{\boldsymbol{H}^{1}\left(\Omega_{0}\right)} \leq C e^{-\sigma_{0} k_{1} R_{t}}\|\boldsymbol{u}\|_{\boldsymbol{H}^{1 / 2}(\Gamma)} .
$$

Proof. Since

$$
\mathcal{A}\left(\widetilde{\boldsymbol{u}}-\widetilde{\boldsymbol{u}}_{t}, \boldsymbol{\phi}\right)=0, \quad \text { for all } \phi \in \boldsymbol{H}_{0}^{1}\left(\Omega_{\infty}\right),
$$

Theorem 5.1, Lemma 5.1, Lemma 5.2 and Theorem 4.4 give

$$
\begin{aligned}
\left\|\widetilde{\boldsymbol{u}}-\widetilde{\boldsymbol{u}}_{t}\right\|_{\boldsymbol{H}^{1}\left(\Omega_{\infty}\right)} & \leq C\|\widetilde{\boldsymbol{u}}\|_{\boldsymbol{H}^{1 / 2}\left(\Gamma_{\infty}\right)} \leq C e^{-\sigma_{0} k_{1} R_{t}}\|\widetilde{\boldsymbol{u}}\|_{\boldsymbol{H}^{1}\left(\Omega_{\infty}\right)} \\
& \leq C e^{-\sigma_{0} k_{1} R_{t}}\|\boldsymbol{u}\|_{\boldsymbol{H}^{1 / 2}(\Gamma)} .
\end{aligned}
$$

Thus (5.11) follows and, since $\widetilde{\boldsymbol{u}}=\boldsymbol{u}$ on $\Omega_{0}$, (5.12) also follows. This completes the proof of the theorem.

\section{Analysis of the Galerkin approximation}

For simplicity, we assume that $\partial \Omega$ is polyhedral and choose $\Gamma_{\infty}$ so that $\Omega_{\infty}$ is also polyhedral. For a triangulation $\mathbb{T}_{h}$ of $\Omega_{\infty}$, let $\widetilde{\boldsymbol{V}}_{h}$ be a finite element space of continuous piecewise polynomial complex valued vector functions which vanish on $\Gamma_{\infty}$. We further simplify by assuming that $\boldsymbol{g}$ coincides with a function in $\widetilde{\boldsymbol{V}}_{h}$ on $\Gamma$. The Galerkin approximation of $\widetilde{\boldsymbol{u}}_{t}$ is the function $\boldsymbol{u}_{h} \in \widetilde{\boldsymbol{V}}_{h}$ satisfying

$$
\begin{aligned}
\mathcal{B}\left(\boldsymbol{u}_{h}, \boldsymbol{\psi}_{h}\right) & =0 \quad \text { for all } \boldsymbol{\psi}_{h} \in \boldsymbol{V}_{h}, \\
\boldsymbol{u}_{h} & =\boldsymbol{g} \text { on } \partial \Omega
\end{aligned}
$$

Here $\boldsymbol{V}_{h}$ denotes the set of functions in $\widetilde{\boldsymbol{V}}_{h}$ which vanish on $\Gamma$ and $\mathcal{B}(\boldsymbol{u}, \boldsymbol{v})$ denotes the scaled form defined by

$$
\mathcal{B}(\boldsymbol{u}, \boldsymbol{v})=\mathcal{A}\left(\boldsymbol{u}, \bar{d}^{-1} \boldsymbol{v}\right) .
$$

We note that Theorem 5.1 is obviously valid for the form $\mathcal{B}$ as well.

Our goal is to apply the so-called "Schatz finite element duality argument [18]" to show that, for sufficiently small mesh size $h$, the solution to (6.1) exists and is unique. Unfortunately, to obtain this result we shall have to put a smallness constraint on our PML function $\tilde{\sigma}$. To this end, we fix $\tilde{\sigma}_{1}$ to be a function satisfying (3.2) with $\sigma_{0}=1$ and set $\tilde{\sigma}=\sigma_{0} \tilde{\sigma}_{1}$. We start by proving a Gårding type inequality for the form $\mathcal{B}(\cdot, \cdot)$.

Lemma 6.1. There exists $S_{\gamma}>0$ and a positive constant $C$ (depending on $S_{\gamma}$ ), such that whenever $\sigma_{0} \leq S_{\gamma}$,

$$
\|\boldsymbol{w}\|_{\boldsymbol{H}^{1}\left(\Omega_{\infty}\right)}^{2} \leq C\left(|\mathcal{B}(\boldsymbol{w}, \boldsymbol{w})|+\|\boldsymbol{w}\|_{\boldsymbol{H}^{1}\left(\Omega_{\infty}\right)}\|\boldsymbol{w}\|_{\boldsymbol{L}^{2}\left(\Omega_{\infty}\right)}\right) \quad \text { for all } \boldsymbol{w} \in \boldsymbol{H}^{1}\left(\Omega_{\infty}\right) .
$$


Proof. The sesquilinear form $\mathcal{A}$ can be rewritten as

$$
\begin{aligned}
\mathcal{A}(\boldsymbol{w}, \boldsymbol{\psi}) & =\sum_{i=1}^{3} \int_{\Omega_{\infty}}\left(\left(\frac{\tilde{d}^{2}}{d} \boldsymbol{P}+d(\boldsymbol{I}-\boldsymbol{P})\right) \boldsymbol{\nabla} \boldsymbol{w}_{i}\right) \cdot \boldsymbol{\nabla} \overline{\boldsymbol{\psi}}_{i} d x \\
& +\gamma \int_{\Omega_{\infty}}\left(\tilde{d}^{2} d\right)^{-1} \nabla \cdot\left(\left(\tilde{d}^{2} \boldsymbol{P}+\tilde{d} d(\boldsymbol{I}-\boldsymbol{P})\right) \boldsymbol{w}\right) \nabla \\
& \cdot\left(\left(\tilde{d}^{2} \boldsymbol{P}+\tilde{d} d(\boldsymbol{I}-\boldsymbol{P})\right) \overline{\boldsymbol{\psi}}\right) d x \\
& -k^{2} \int_{\Omega_{\infty}} \tilde{d}^{2} d \boldsymbol{w} \cdot \overline{\boldsymbol{\psi}} d x .
\end{aligned}
$$

Let $\boldsymbol{D}$ denote the matrix $(\tilde{d} / d-1) \boldsymbol{P}$. Using the equalities

$$
\left(\frac{\tilde{d}^{2}}{d} \boldsymbol{P}+d(\boldsymbol{I}-\boldsymbol{P})\right)=d\left(\left(\frac{\tilde{d}}{d}+1\right) \boldsymbol{D}+\boldsymbol{I}\right) \text { and }\left(\tilde{d}^{2} \boldsymbol{P}+\tilde{d} d(\boldsymbol{I}-\boldsymbol{P})\right)=\tilde{d} d(\boldsymbol{D}+\boldsymbol{I})
$$

gives

$$
\begin{aligned}
\mathcal{B}(\boldsymbol{w}, \boldsymbol{\psi}) & =\sum_{i=1}^{3} \int_{\Omega_{\infty}}\left(\left(\left(\frac{\tilde{d}}{d}+1\right) \boldsymbol{D}+\boldsymbol{I}\right) \boldsymbol{\nabla} \boldsymbol{w}_{i}\right) \cdot \boldsymbol{\nabla} \overline{\boldsymbol{\psi}}_{i} d x \\
& +\gamma \int_{\Omega_{\infty}}(\nabla \cdot(\boldsymbol{D}+\boldsymbol{I}) \boldsymbol{w})(\nabla \cdot(\boldsymbol{D}+\boldsymbol{I}) \overline{\boldsymbol{\psi}}) d x+\text { L.O. }
\end{aligned}
$$

Here we have used the notation "L.O." to denote terms which have at least one undifferentiated component of $\boldsymbol{w}$ or $\boldsymbol{\psi}$ so that

$$
\mid \text { L.O. } \mid \leq C\left(\|\boldsymbol{w}\|_{\boldsymbol{H}^{1}\left(\Omega_{\infty}\right)}\|\boldsymbol{\psi}\|_{\boldsymbol{L}^{2}\left(\Omega_{\infty}\right)}+\|\boldsymbol{\psi}\|_{\boldsymbol{H}^{1}\left(\Omega_{\infty}\right)}\|\boldsymbol{w}\|_{\boldsymbol{L}^{2}\left(\Omega_{\infty}\right)}\right) .
$$

A key point in our proof will be the fact that we can make various quantities involving $\boldsymbol{D}$ arbitrarily small by decreasing $\sigma_{0}$. We note that the coefficient $(\tilde{d} / d-1)$ is supported only in the transition region and that

$$
\left|\frac{\tilde{d}}{d}-1\right| \leq C \sigma_{0}
$$

Indeed,

$$
\left|\frac{\tilde{d}}{d}-1\right|=\left|\frac{1+i \tilde{\sigma}}{1+i \sigma}-1\right|=\left|\frac{i(\tilde{\sigma}-\sigma)}{1+i \sigma}\right| \leq \sigma_{0} \max _{r}\left|\tilde{\sigma}_{1}(r)-\sigma_{1}(r)\right| .
$$

We clearly have

$$
\begin{aligned}
\sum_{i=1}^{3} \int_{\Omega_{\infty}} & \left(\left(\left(\frac{\tilde{d}}{d}+1\right) \boldsymbol{D}+\boldsymbol{I}\right) \boldsymbol{\nabla} \boldsymbol{w}_{i}\right) \cdot \boldsymbol{\nabla} \overline{\boldsymbol{\psi}}_{i} d x \\
& =\sum_{i=1}^{3} \int_{\Omega_{\infty}} \boldsymbol{\nabla} \boldsymbol{w}_{i} \cdot \boldsymbol{\nabla} \overline{\boldsymbol{\psi}}_{i} d x+\sum_{i=1}^{3} \int_{\Omega_{\infty}}\left(\left(\left(\frac{\tilde{d}}{d}+1\right) \boldsymbol{D}\right) \boldsymbol{\nabla} \boldsymbol{w}_{i}\right) \cdot \boldsymbol{\nabla} \overline{\boldsymbol{\psi}}_{i} d x
\end{aligned}
$$

In addition,

$$
\nabla \cdot(\boldsymbol{D} \boldsymbol{w})=\operatorname{tr}(\boldsymbol{D} \nabla \boldsymbol{w})+\operatorname{div} \boldsymbol{D}^{t} \cdot \boldsymbol{w}
$$


where the divergence $\operatorname{div} \boldsymbol{D}^{t}$ of the matrix function $\boldsymbol{D}^{t}$ is defined to be the vector whose components are the divergences of the rows of $\boldsymbol{D}^{t}$. We can then rewrite

$$
\begin{aligned}
\gamma \int_{\Omega_{\infty}}(\nabla \cdot(\boldsymbol{D}+\boldsymbol{I}) \boldsymbol{w})(\nabla \cdot(\boldsymbol{D}+\boldsymbol{I}) \overline{\boldsymbol{\psi}}) d x \\
=\gamma \int_{\Omega_{\infty}} \nabla \cdot \boldsymbol{w} \nabla \cdot \overline{\boldsymbol{\psi}} d x+\gamma \int_{\Omega_{\infty}} \operatorname{tr}(\boldsymbol{D} \nabla \boldsymbol{w}) \operatorname{tr}(\boldsymbol{D} \nabla \overline{\boldsymbol{\psi}}) d x \\
+\gamma \int_{\Omega_{\infty}} \operatorname{tr}(\boldsymbol{D} \nabla \boldsymbol{w}) \nabla \cdot \overline{\boldsymbol{\psi}} d x+\gamma \int_{\Omega_{\infty}} \nabla \cdot \boldsymbol{w} \operatorname{tr}(\boldsymbol{D} \nabla \overline{\boldsymbol{\psi}}) d x \\
+ \text { L.O. }
\end{aligned}
$$

Combining (6.3)-(6.8) and applying the Schwarz and arithmetic-geometric mean inequalities gives

$$
\mathcal{B}(\boldsymbol{w}, \boldsymbol{w}) \geq a(\boldsymbol{w}, \boldsymbol{w})-C_{\gamma} \sigma_{0} a(\boldsymbol{w}, \boldsymbol{w})-C_{0}\|\boldsymbol{w}\|_{\boldsymbol{H}^{1}\left(\Omega_{\infty}\right)}\|\boldsymbol{w}\|_{\boldsymbol{L}^{2}\left(\Omega_{\infty}\right)} .
$$

Here

$$
a(\boldsymbol{w}, \boldsymbol{\psi})=\sum_{i=1}^{3}\left(\boldsymbol{\nabla} \boldsymbol{w}_{i}, \boldsymbol{\nabla} \boldsymbol{\psi}_{i}\right)+\gamma(\nabla \cdot \boldsymbol{w}, \nabla \cdot \boldsymbol{\psi})+(\boldsymbol{w}, \boldsymbol{\psi}) .
$$

The lemma immediately follows, taking $S_{\gamma}<1 / C_{\gamma}$.

Remark 6.1. It should be noted that, in addition to being independent of $\sigma_{0}$, the constants $C_{\gamma}$ and $C_{0}$ (and consequently $S_{\gamma}$ and $C$ ) in the proof above are also independent of the diameter of the region $\Omega_{\infty}$.

In order to apply the duality argument, we shall also need the following regularity result.

Proposition 6.1. There exists an $s>\frac{1}{2}$ and a constant $C_{\text {reg }}>0$ such that for any $\boldsymbol{f} \in \boldsymbol{L}^{2}\left(\Omega_{\infty}\right)$, the solution $\boldsymbol{w} \in \boldsymbol{H}_{0}^{1}\left(\Omega_{\infty}\right)$ of

$$
\mathcal{B}(\boldsymbol{\psi}, \boldsymbol{w})=(\boldsymbol{\psi}, \boldsymbol{f}) \text { for all } \boldsymbol{\psi} \in \boldsymbol{H}_{0}^{1}\left(\Omega_{\infty}\right)
$$

is in $\boldsymbol{H}^{1+s}\left(\Omega_{\infty}\right)$ and satisfies

$$
\|\boldsymbol{w}\|_{\boldsymbol{H}^{1+s}\left(\Omega_{\infty}\right)} \leq C_{r e g}\|\boldsymbol{f}\|_{\boldsymbol{L}^{2}\left(\Omega_{\infty}\right)} .
$$

Remark 6.2. Full interior regularity of the solution $\boldsymbol{w}$ to this problem follows from the $C^{2}$ smoothness of the PML coefficients. Regularity near the boundary follows from known results for the regularity of solutions to the constant coefficient equation (2.1) on polyhedral domains (see for example Theorems 2.3 (2D) and 4.5 (3D) of [13]).

We are now ready to state the main result of this section, i.e., the unique solvability for the Galerkin approximation.

Theorem 6.1. Assume that $\sigma_{0} \leq S_{\gamma}$. There exists an $h_{0}>0$ such that whenever $h<h_{0}$, there is a unique solution $\boldsymbol{u}_{h} \in \widetilde{\boldsymbol{V}}_{h}$ of (6.1) and

$$
\left\|\widetilde{\boldsymbol{u}}_{t}-\boldsymbol{u}_{h}\right\|_{\boldsymbol{H}^{1}\left(\Omega_{\infty}\right)} \leq C \inf \left\|\widetilde{\boldsymbol{u}}_{t}-\boldsymbol{v}_{h}\right\|_{\boldsymbol{H}^{1}\left(\Omega_{\infty}\right)} .
$$

The infimum above is taken over $\boldsymbol{v}_{h} \in \widetilde{\boldsymbol{V}}_{h}$ with $\boldsymbol{v}_{h}=\boldsymbol{g}$ on $\Gamma$. 
Proof. Given Lemma 6.1 and Proposition 6.1 the theorem essentially follows from the well known argument given by Schatz ([18]), which we sketch. For $\boldsymbol{w} \in$ $\boldsymbol{H}_{0}^{1}\left(\Omega_{\infty}\right)$, let $\boldsymbol{w}_{h} \in \boldsymbol{V}_{h}$ be a solution to

$$
\mathcal{B}\left(\boldsymbol{w}_{h}, \boldsymbol{\theta}\right)=\mathcal{B}(\boldsymbol{w}, \boldsymbol{\theta}) \text { for all } \boldsymbol{\theta} \in \boldsymbol{V}_{h}
$$

By finite element duality [14] and Proposition 6.1, one shows that $\boldsymbol{e}_{h} \equiv \boldsymbol{w}-\boldsymbol{w}_{h}$ satisfies

$$
\left\|\boldsymbol{e}_{h}\right\|_{\boldsymbol{L}^{2}\left(\Omega_{\infty}\right)} \leq C h^{s}\left\|\boldsymbol{e}_{h}\right\|_{\boldsymbol{H}^{1}\left(\Omega_{\infty}\right)} .
$$

It follows from Lemma 6.1 that for $h$ sufficiently small,

$$
\left\|\boldsymbol{e}_{h}\right\|_{\boldsymbol{H}^{1}\left(\Omega_{\infty}\right)}^{2} \leq C \mathcal{B}\left(\boldsymbol{e}_{h}, \boldsymbol{e}_{h}\right)
$$

Taking $\boldsymbol{w}=\mathbf{0}$ implies that the (square) discrete system has unique solutions from which existence also follows. Finally, the error estimate is an easy consequence of (6.10) and Galerkin orthogonality.

\section{Computational Results}

We illustrate the performance of the PML technique on a problem in the case of two spatial dimensions. We rig up a problem with known solution. Specifically, we take $\gamma=3$ and $k=1$ in equation (2.1) and consider the function

$$
\boldsymbol{u}(x, y)=\boldsymbol{u}(r, \theta)=\nabla \times\left(h_{1}^{1}(k r) \cos (\theta)\right)+\nabla\left(h_{1}^{1}\left(k_{1} r\right) \cos (\theta)\right)
$$

It is clear that the above function satisfies (2.1)-(2.5). The first term is solenodial while the second is irrotational, and so both wave components are present.

We now consider approximating the solution of (2.1) with $\Omega=[-1,1]^{2}$ and $\boldsymbol{g}=\boldsymbol{u}$ (given by (7.1)) on $\Gamma$. By construction, the solution is just the function $\boldsymbol{u}$ given by (7.1).

To define the PML approximation, we take $r_{0}=3, r_{1}=4$ and $\sigma_{0}=1$. We truncate the domain so that $\Omega_{\infty}=(-5,5)^{2} \backslash[-1,1]^{2}$. Although it is not clear that this choice of $\sigma_{0}$ satisfies the smallness assumption of our theorem, it nevertheless appears to work (as we shall see below). In all of our reported experiments, we shall compare the difference between the Galerkin solution $\boldsymbol{u}_{h}$ defined by (6.1) and $\boldsymbol{u}$ defined above.

Figure 1 gives a surface plot representation for the real part of the second component of the exact solution and the finite element PML approximation. As suggested by the theory, the PML solution appears to be close to the exact solution in the inside of the PML layer and goes to zero quickly in the PML region. The effect of the transition region is further illustrated from the overhead view given in Figure2, where we can clearly see the "cut-off" annulus $B_{4} \backslash \overline{B_{3}}$. 

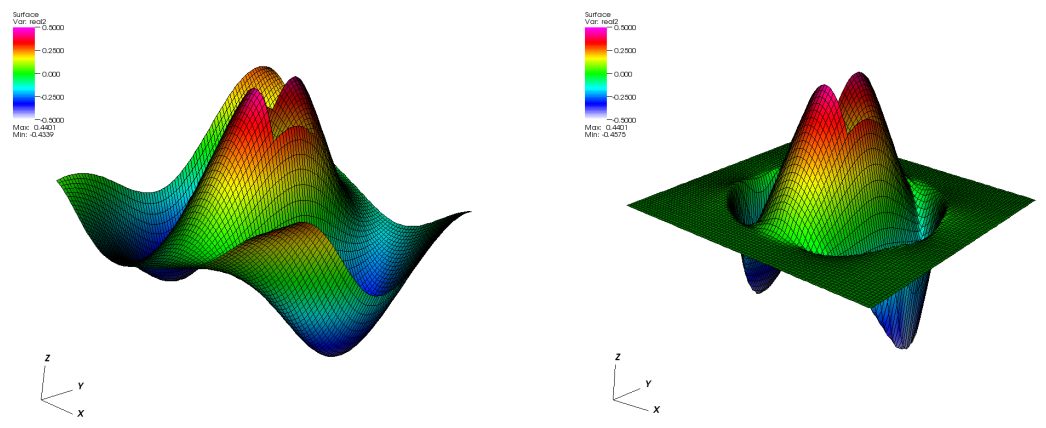

Figure 1. The real part of the second component in the exact and the PML solutions
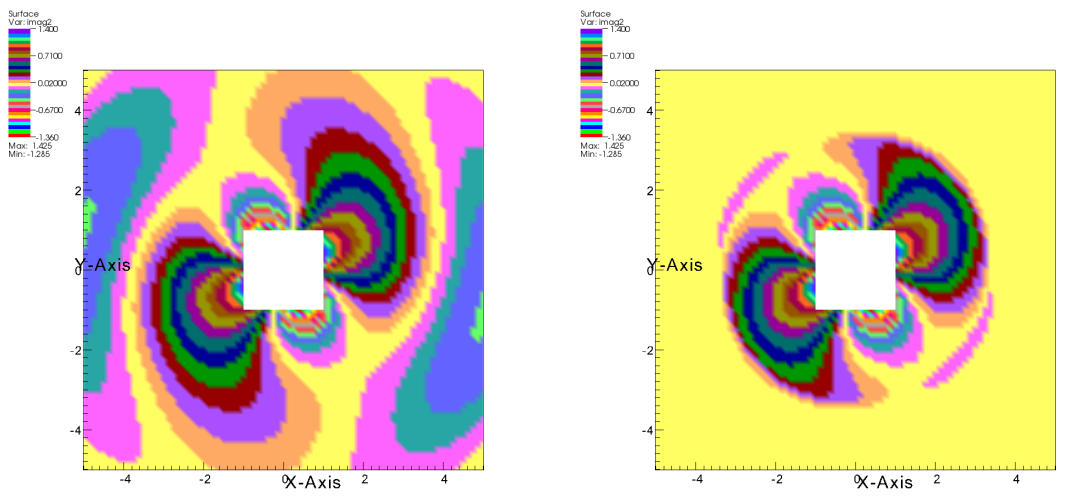

Figure 2. The imaginary part of the second component in the exact and the PML solutions

To more precisely gauge the behavior of the method, we compute the norms of the error between $\boldsymbol{u}$ and $\boldsymbol{u}_{h}$ near $\partial \Omega$ (of course, this is the only meaningful computation, as $\boldsymbol{u}$ and $\boldsymbol{u}_{h}$ are significantly different in the PML region). Specifically, we report errors on $\Omega_{*} \equiv(-2,2)^{2} \backslash[-1,1]^{2}$. Table 7.1 gives the $\boldsymbol{L}^{2}\left(\Omega_{*}\right)$ and $\boldsymbol{H}^{1}\left(\Omega_{*}\right)$ errors as a function of the mesh size. The $\boldsymbol{H}^{1}\left(\Omega_{*}\right)$ results clearly exhibit the expected first order of convergence. The $\boldsymbol{L}^{2}\left(\Omega_{*}\right)$ results show a convergence rate less than second order, which is consistent with the fact that the domain $\Omega_{\infty}$ has re-entrant corners.

It is interesting to note that in the above calculations we have not yet seen the pollution effect of the domain truncation come into play. This is not surprising, as this error is exponentially small in the size of the domain and it appears that we have not yet made $h$ small enough to see its effect. 
TABLE 7.1. $L^{2}-$ and $H^{1}-$ norms of the error

\begin{tabular}{|c|r|r|r|}
\hline$h$ & \# dofs & $\boldsymbol{L}^{2}\left(\Omega_{*}\right)$-error & $\boldsymbol{H}^{1}\left(\Omega_{*}\right)$-error \\
\hline$\frac{1}{2}$ & 1728 & 0.384539 & 1.990330 \\
\hline$\frac{1}{4}$ & 6528 & 0.274241 & 1.025252 \\
\hline$\frac{1}{8}$ & 25344 & 0.092068 & 0.482071 \\
\hline$\frac{1}{16}$ & 99840 & 0.029358 & 0.232628 \\
\hline$\frac{1}{32}$ & 396288 & 0.010079 & 0.114665 \\
\hline
\end{tabular}

\section{Appendix A}

In this appendix, we prove the identities (3.4), (3.5) and (3.6) by a complex variable analyticity argument. To do this, as in the proof of Theorem 4.1, we replace $i$ by $z$ in the definition of the PML operators for $z \in \widetilde{\mathbb{C}}$. The main parts of the arguments are standard and we only sketch them here.

We first prove (3.4). Fix $\boldsymbol{w} \in H_{l o c}^{2}\left(\mathbb{R}^{3}\right)$ and $\phi \in C_{0}^{\infty}\left(\mathbb{R}^{3}\right)$. For $z \in \widetilde{\mathbb{C}}$, we set

$$
F(z)=\int_{\mathbb{R}^{3}} \widetilde{d}^{2} d(\widetilde{\boldsymbol{\Delta}} \boldsymbol{w}+\widetilde{\nabla} \times \widetilde{\nabla} \times \boldsymbol{w}-\widetilde{\nabla} \widetilde{\nabla} \cdot \boldsymbol{w}) \cdot \boldsymbol{\phi} d x .
$$

To show that $F$ is analytic, we apply the dominated convergence theorem to the difference quotients. That the difference quotients can be dominated by an integrable function follows from the properties of $\widetilde{\sigma}$, namely $\widetilde{\sigma}, \sigma, \widetilde{d}, d, \widetilde{d}{ }^{-1}, d^{-1} \in L^{\infty}(\mathbb{R})$. Next, for real $s \in \widetilde{\mathbb{C}}$, a change of variables gives

$$
F(s)=\int_{\mathbb{R}^{3}}\left[\boldsymbol{\Delta} \boldsymbol{w}_{s}+\boldsymbol{\nabla} \times \boldsymbol{\nabla} \times \boldsymbol{w}_{s}-\nabla \nabla \cdot \boldsymbol{w}_{s}\right] \cdot \boldsymbol{\phi}_{s} d x .
$$

Here $\boldsymbol{w}_{s}(\boldsymbol{x})=\boldsymbol{w}\left(T_{s}^{-1} \boldsymbol{x}\right)$ and $\boldsymbol{\phi}_{s}(\boldsymbol{x})=\boldsymbol{\phi}\left(T_{s}^{-1} \boldsymbol{x}\right)$. This is because $\widetilde{\sigma} \in C^{2}(\mathbb{R}), \boldsymbol{w}_{s} \in$ $H_{l o c}^{2}\left(\mathbb{R}^{3}\right)$ and the bracketed quantity in (8.1) vanishes. It follows that $F(z)=0$ identically. As this holds for all $\phi \in C_{0}^{\infty}\left(\mathbb{R}^{3}\right)$, (3.4) immediately follows.

Remark 8.1. It is possible but tedious to verify (3.4) directly. The complex variable viewpoint simplifies this calculation as well as the dervation of the remaining two identities.

For (3.5), we fix $\boldsymbol{w} \in H^{2}\left(\mathbb{R}^{3}\right)$ and $\phi \in \boldsymbol{C}_{0}^{\infty}\left(\mathbb{R}^{3}\right)$ and set

$$
F(z)=\int_{\mathbb{R}^{3}} \widetilde{d}^{2} d(\widetilde{\boldsymbol{\Delta}} \boldsymbol{w}) \cdot(\widetilde{\boldsymbol{\nabla}} \phi) d x-\int_{\mathbb{R}^{3}} \widetilde{d}^{2} d(\widetilde{\boldsymbol{\nabla}}(\widetilde{\nabla} \cdot \boldsymbol{w})) \cdot(\widetilde{\boldsymbol{\nabla}} \phi) d x .
$$

The analyticity of $F$ is concluded as above. Changing variables for real $s \in \widetilde{\mathbb{C}}$ gives

$$
F(s)=\int_{\mathbb{R}^{3}}\left(\boldsymbol{\Delta} \boldsymbol{w}_{s}\right) \cdot\left(\nabla \phi_{s}\right) d x-\int_{\mathbb{R}^{3}}\left(\nabla\left(\nabla \cdot \boldsymbol{w}_{s}\right)\right) \cdot\left(\nabla \phi_{s}\right) d x .
$$

That the two integrals in $F(s)$ are equal follows by integration by parts, first moving derivatives from $\boldsymbol{w}_{s}$ to $\phi_{s}$ and subsequently moving derivatives from $\phi_{s}$ back to $\boldsymbol{w}_{s}$. This makes sense since $\phi_{s} \in C^{2}\left(\mathbb{R}^{3}\right)$ and has compact support. Thus, $F(s)=0$ extends to $F(z)=0$. This identity extends to $\phi \in H^{1}\left(\mathbb{R}^{3}\right)$ by density.

The argument for showing (3.6) is completely analogous to that used for (3.5) and will be omitted. 
Remark 8.2. The proofs critically depend on the smoothness of $\widetilde{\sigma}$, i.e., $\widetilde{\sigma} \in C^{2}(\mathbb{R})$. It is by no means clear if these results hold for less smooth $\widetilde{\sigma}$, e.g., $\widetilde{\sigma}$ leading to piecewise constant $\sigma$.

\section{Appendix B}

In order to prove Lemma 5.2 we first state two propositions. The first is a classical interior estimate for the solution of an elliptic equation whose proof is elementary. The second was proved in [2]. Note that, in this section, we use additional expanding domains $\Omega_{i}, i=3, \ldots, 6$, corresponding to $r_{2}<r_{3}<\cdots<r_{6}$ in this section.

Proposition 9.1. Suppose that $w$ satisfies the equation

$$
\Delta w+\beta w=0
$$

and $\boldsymbol{v}$ satisfies the equation

$$
\boldsymbol{\Delta} \boldsymbol{v}+\gamma \boldsymbol{\nabla} \nabla \cdot \boldsymbol{v}+\beta \boldsymbol{v}=\mathbf{0}
$$

in a domain $D$ with a (possibly complex) constant $\beta$. If $D_{1}$ is a subdomain whose closure is contained in $D$, then

$$
\|w\|_{H^{2}\left(D_{1}\right)} \leq C\|w\|_{L^{2}(D)}
$$

and

$$
\|\boldsymbol{v}\|_{H^{1}\left(D_{1}\right)} \leq C\|\boldsymbol{v}\|_{L^{2}(D)} .
$$

Proposition 9.2. Assume that $w$ is bounded at infinity and satisfies (9.1) in $\Omega^{c} \backslash \bar{\Omega}_{1}$ with $\beta=k^{2} d_{0}^{2}$ or $\beta=k_{1}^{2} d_{0}^{2}$. Set $S_{\alpha}=\left\{\boldsymbol{x}: \operatorname{dist}\left(\boldsymbol{x}, \Gamma_{\infty}\right)<\alpha\right\}$ with $\alpha$ fixed independent of $R_{t}>r_{6}$ and small enough that $\bar{S}_{\alpha}$ is in $\Omega^{c} \backslash \bar{\Omega}_{6}$. Then

$$
\|w\|_{L^{2}\left(S_{\alpha}\right)} \leq C e^{-\sigma_{0} k_{1} R_{t}}\|w\|_{L^{2}\left(\Omega_{5} \backslash \bar{\Omega}_{3}\right)} .
$$

Proof of Lemma 5.2. We use the decomposition given in (2.3). As seen in 3], $\phi \equiv k_{1}^{-2} \nabla \cdot \boldsymbol{u}$ is a scalar potential for the irrotational part $\boldsymbol{\psi}$, and it, along with each Cartesian component of $\boldsymbol{\psi}=\nabla \phi$, satisfies (9.1) with $\beta=k_{1}^{2} d_{0}^{2}$. Hence by Proposition 9.1, Proposition 9.2 and a trace inequality, we see that

$$
\begin{aligned}
\|\boldsymbol{\psi}\|_{\boldsymbol{H}^{1 / 2}\left(\Gamma_{\infty}\right)} & \leq C\|\boldsymbol{\psi}\|_{\boldsymbol{H}^{1}\left(S_{\alpha / 2}\right)} \leq C\|\boldsymbol{\psi}\|_{\boldsymbol{L}^{2}\left(S_{\alpha}\right)} \leq C e^{-\sigma_{0} k_{1} R_{t}}\|\boldsymbol{\psi}\|_{\boldsymbol{L}^{2}\left(\Omega_{5} \backslash \bar{\Omega}_{3}\right)} \\
& \leq C e^{-\sigma_{0} k_{1} R_{t}}\|\phi\|_{H^{1}\left(\Omega_{5} \backslash \bar{\Omega}_{3}\right)} \leq C e^{-\sigma_{0} k_{1} R_{t}}\|\phi\|_{L^{2}\left(\Omega_{6} \backslash \bar{\Omega}_{2}\right)} \\
& \leq C e^{-\sigma_{0} k_{1} R_{t}}\|\nabla \cdot \boldsymbol{u}\|_{L^{2}\left(\Omega_{6} \backslash \bar{\Omega}_{2}\right)} \leq C e^{-\sigma_{0} k_{1} R_{t}}\|\boldsymbol{u}\|_{\boldsymbol{L}^{2}\left(\Omega_{\infty}\right)} .
\end{aligned}
$$

For the last inequality we used the second part of Proposition 9.1 since $\boldsymbol{u}$ satisfies (9.2) with $\beta=k^{2} d_{0}^{2}$. Similarly, each Cartesian component of $\boldsymbol{\zeta}$ satisfies (9.1) with $\beta=k^{2} d_{0}^{2}$, so that by Proposition 9.1, Proposition 9.2 and a trace inequality we have

$$
\begin{aligned}
\|\boldsymbol{\zeta}\|_{\boldsymbol{H}^{1 / 2}\left(\Gamma_{\infty}\right)} & \leq C\|\boldsymbol{\zeta}\|_{\boldsymbol{H}^{1}\left(S_{\alpha / 2}\right)} \leq C\|\boldsymbol{\zeta}\|_{\boldsymbol{L}^{2}\left(S_{\alpha}\right)} \leq C e^{-\sigma_{0} k R_{t}}\|\boldsymbol{\zeta}\|_{\boldsymbol{L}^{2}\left(\Omega_{5} \backslash \bar{\Omega}_{3}\right)} \\
& \leq C e^{-\sigma_{0} k R_{t}}\left(\|\boldsymbol{\psi}\|_{\boldsymbol{L}^{2}\left(\Omega_{5} \backslash \bar{\Omega}_{3}\right)}+\|\boldsymbol{u}\|_{\boldsymbol{L}^{2}\left(\Omega_{5}\right)} \leq C e^{-\sigma_{0} k R_{t}}\|\boldsymbol{u}\|_{\boldsymbol{L}^{2}\left(\Omega_{\infty}\right)} .\right.
\end{aligned}
$$

Combining (9.5) and (9.6), and noting that $k_{1}<k$, completes the proof of Lemma 5.2 . 


\section{ACKNOWLEDGMENTS}

This work was supported in part by the National Science Foundation through Grant DMS-0609544 and in part by award number KUS-C1-016-04 made by King Abdulla University of Science and Technology (KAUST).

\section{REFERENCES}

[1] J.-P. Bérenger. A perfectly matched layer for the absorption of electromagnetic waves. J. Comput. Phys., 114(2):185-200, 1994. MR.1294924 (95e:78002)

[2] J. H. Bramble and J. E. Pasciak. Analysis of a finite PML approximation for the three dimensional time-harmonic Maxwell and acoustic scattering problems. Math. Comp., 76(258):597614 (electronic), 2007. MR2291829 (2008b:65130)

[3] J. H. Bramble and J. E. Pasciak. A note on the existence and uniqueness of solutions of frequency domain elastic wave problems: A priori estimates in $H^{1}$. J. Math. Anal. Appl., 345:396-404, 2008. MR2422659(2009j:74053)

[4] W. Chew and Q. H. Liu. Perfectly matched layers for elastodynamics: A new absorbing boundary condition. J. Comput. Acoust., 4(4):341-359, 1996.

[5] W. Chew and W. Weedon. A 3d perfectly matched medium for modified Maxwell's equations with streched coordinates. Microwave Opt. Techno. Lett., 13(7):599-604, 1994.

[6] F. Collino and P. Monk. The perfectly matched layer in curvilinear coordinates. SIAM J. Sci. Comp., 19(6):2061-2090, 1998. MR1638033(99e:78029)

[7] F. Collino and C. Tsogka. Application of the perfectly matched absorbing layer model to the linear elastodynamic problem in anisotropic heterogeneous media. Geophysics, 66(1):294-307, 2001.

[8] D. Colton and R. Kress. Inverse acoustic and electromagnetic scattering theory, volume 93 of Applied Mathematical Sciences. Springer-Verlag, Berlin, second edition, 1998. MR.1635980 (99c:35181)

[9] F. D. Hastings, J. B. Schneider, and S. L. Broschat. Application of the perfectly matched layer (pml) absorbing boundary condition to elastic wave propagation. The Journal of the Acoustical Society of America, 100(5):3061-3069, 1996.

[10] M. Lassas and E. Somersalo. On the existence and convergence of the solution of PML equations. Computing, 60(3):229-241, 1998. MR1621305 (99a:65133)

[11] P. Monk. Finite element methods for Maxwell's equations. Numerical Mathematics and Scientific Computation. Oxford University Press, New York, 2003. MR2059447 (2005d:65003)

[12] J.-C. Nédélec. Acoustic and electromagnetic equations, volume 144 of Applied Mathematical Sciences. Springer-Verlag, New York, 2001. Integral representations for harmonic problems. MR1822275 (2002c:35003)

[13] S. Nicaise. About the Lamé system in a polygonal or a polyhedral domain and a coupled problem between the Lamé system and the plate equation. I. Regularity of the solutions. Ann. Scuola Norm. Sup. Pisa Cl. Sci. (4), 19(3):327-361, 1992. MR1205404(94b:35069)

[14] J. Nitsche. Ein Kriterium für die Quasi-Optimalität des Ritzschen Verfahrens. Numer. Math., 11:346-348, 1968. MR0233502 (38:1823)

[15] J. Peetre. Espaces d'interpolation et théorèm de Soboleff. Ann. Inst. Fourier, 16:279-317, 1966. MR0221282 (36:4334)

[16] C. J. Randall. Absorbing boundary condition for the elastic wave equation: Velocity-stress formulation. Geophysics, 54(9):1141-1152, 1989.

[17] M. Renardy and R. C. Rogers. An introduction to partial differential equations, volume 13 of Texts in Applied Mathematics. Springer-Verlag, New York, second edition, 2004. MR 2028503 (2004j:35001)

[18] A. Schatz. An observation concerning Ritz-Galerkin methods with indefinite bilinear forms. Math. Comp., 28:959-962, 1974. MR0373326 (51:9526)

[19] L. Tartar. Topics in Nonlinear Analysis. Math. d'Orsay, Univ. Paris-Sud, 1978. MR532371 (81b:35001)

[20] Y. Zheng and X. Huang. Anisotropic perfectly matched layers for elastic waves in cartesian and curvilinear coordinates. Research report, Massachusetts Institute of Technology, 2002. 
Department of Mathematics, Texas A\&M University, College Station, Texas 778433368

E-mail address: bramble@math.tamu.edu

Department of Mathematics, Texas A\&M University, College Station, Texas 778433368

E-mail address: pasciak@math.tamu.edu

Department of Mathematics, Texas A\&M University, College Station, Texas 778433368

E-mail address: trenev@math.tamu.edu 